\title{
FTIR Microspectroscopy of Polymeric Systems
}

\author{
Rohit Bhargava $^{1}$, Shi-Qing Wang ${ }^{2}$, Jack L. Koenig ${ }^{3}$ \\ ${ }^{1}$ Laboratory of Chemical Physics, NIDDK, National Institutes of Health, Bethesda, MD \\ 20892, USA, E-mail:rohit@sunder.niddk.nih.gov \\ 2 Department of Polymer Science, University of Akron, Akron, OH, USA, \\ E-mail:wang@polymer.uakron.edu \\ ${ }^{3}$ Department of Macromolecular Science, Case Western Reserve University, Cleveland OH \\ 44106, USA, E-mail: jlk6@po.cwru.edu
}

\begin{abstract}
Fourier transform infrared (FTIR) spectroscopy is a mature analytical technique employed to examine polymeric materials. With the coupling of an infrared interferometer to a microscope equipped with specialized detectors, FTIR spectroscopy has been employed widely to examine microscopic areas in polymers for the last twenty years. Following the emergence of instrumentation techniques that employ focal plane array (FPA) detectors, FTIR microspectroscopy has experienced a recent renaissance in terms of the capability of instrumentation and visualization afforded for examining multicomponent polymer systems. We present an overview of the principles of instrumental configurations used to achieve spatially resolved spectral information, their relative advantages and limitations. Illustrative examples are presented that demonstrate the capabilities of FTIR microspectroscopy and the insight this technique provides into the composition, formation, and behavior of polymeric materials. Finally, some emerging techniques that may permit microspectroscopic analyses on a different spatial scale are reviewed.
\end{abstract}

$1 \quad$ Introduction . . . . . . . . . . . . . . . . 139

2 FTIR Microspectroscopy Using a Single Element Detector . . . . . 140

$2.1 \quad$ Introduction $\ldots \ldots \ldots \ldots \ldots$. . . . . . . . . . . . . . . . . . .

2.2 Instrumentation . . . . . . . . . . . . . . . . 141

2.2.1 Sampling Techniques . . . . . . . . . . . . . . . 142

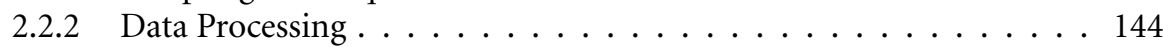

2.2.3 Errors in FTIR Mapping . . . . . . . . . . . . . . . . . . . 145

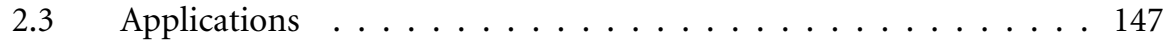

2.3.1 Microsamples, Additives, Contaminants and Degradation . . . . . . 147

2.3.2 Single Polymer Fibers . . . . . . . . . . . . . . . . . . . 150

2.3.3 Polymers in Biological Areas . . . . . . . . . . . . . . . . . . . . . . . . . . . . . . . . . . .

2.3.4 Semi-crystalline Polymers . . . . . . . . . . . . . . 151

2.3.5 Phase Separated Polymer Blends . . . . . . . . . . . . . . . . . . . . 152

2.3.6 Multilayered Polymer Systems . . . . . . . . . . . . . . . . . . . . . . . . . . . . . . . . . . . . . . .

2.3 .7 Diffusion . . . . . . . . . . . . . . . . 155

2.3 .8 Local Orientation . . . . . . . . . . . . . . . . . . . 156

2.3.9 Polymer Surfaces and Interfaces . . . . . . . . . . . . . . . 157 
2.3.10 Polymer-Liquid Crystal Systems . . . . . . . . . . . . . . . . . . . . . . . . . . . . . . . . . . .

2.3.11 Filled Systems . . . . . . . . . . . . . . . . . . . . . 159

2.3.11.1 Carbon Black, Silica and Inorganics Filled Systems . . . . . . . . 159

2.3.11.2 Fibers and Fillers . . . . . . . . . . . . . . . . 160

$2.4 \quad$ Summary ........................... 161

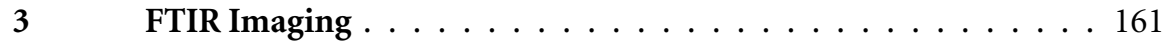

$3.1 \quad$ Introduction . . . . . . . . . . . . . . . . 161

3.1.1 Mapping Versus Imaging . . . . . . . . . . . . . . . . . . . . . . . . . . . . . . . .

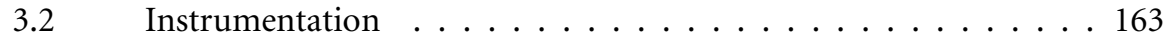

3.2.1 Sampling Techniques . . . . . . . . . . . . . . . . 165

3.2 .2 Sources of Error . . . . . . . . . . . . . . . . . . 165

3.2.2.1 Interferometer Noise . . . . . . . . . . . . . . . . . . . . . . . . . . . . . .

3.2.2.2 Detector Noise . . . . . . . . . . . . . . . . 166

3.2.2.3 Failing Bump Bonds . . . . . . . . . . . . . . . . 166

3.2.2.4 Intensity Distribution, Pixel Saturation and Dynamic Range . . . . 167

3.2.2.5 Sample . . . . . . . . . . . . . . . . . . . 167

3.2.3 Post-Collection Operations . . . . . . . . . . . . . 167

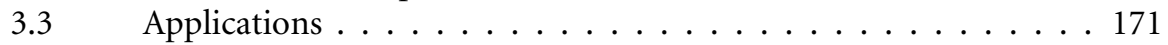

3.3.1 Contaminants/Defects in Polymers . . . . . . . . . . . . 171

3.3.2 Amorphous Polymer Blends . . . . . . . . . . . . . . . . 172

3.3.3 Semi-Crystalline Polymers and Their Blends . . . . . . . . . . . . 172

3.3.4 Polymer Laminate Films . . . . . . . . . . . . . . . . . . . . . . . . . . . . . . . . . . . . . . . . . .

3.3 .5 Polymer-Liquid Systems . . . . . . . . . . . . . . . . . . . . . . . . . . . . . . . . . . . .

3.3.6 Embedded Polymer Systems . . . . . . . . . . . . . . . . . . . . . . . . . . . . . . . . . . . . .

3.3.7 Polymer-Liquid Crystal Systems . . . . . . . . . . . . . . . . . . . . . . . . . . . . . . . . . . .

3.3.8 Phase Separation in Polymers . . . . . . . . . . . . . . . . . 177

3.4 Summary ........................ 178

4 Other Infrared Microspectroscopy Approaches . . . . . . . . . . 179

4.1 Hadamard Transform Infrared Microscopy . . . . . . . . . . . . 179

4.2 Synchrotron Infrared Microscopy . . . . . . . . . . . . . . . 180

4.3 Solid State Focal Plane Array Imaging . . . . . . . . . . . . . . 180

4.4 Scanning Probe Microspectroscopy or Photothermal Imaging . . . 182

$4.5 \quad$ Near Field Infrared Mapping . . . . . . . . . . . . . . . . . 183

4.6 Infrared Diode-Based Imaging $\ldots \ldots \ldots \ldots$

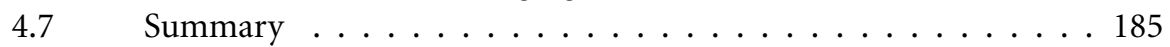

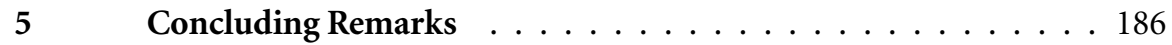

$5.1 \quad$ Instrumental Directions . . . . . . . . . . . . . . 186

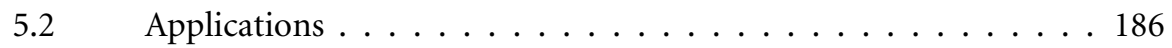

5.3 Processing Techniques and Strategies . . . . . . . . . . . 187 
Summary .......................... 187

References . . . . . . . . . . . . . . . . . . . . . . 188

1

\section{Introduction}

Most utility polymeric articles available today contain multiphase polymeric systems comprised of semi-crystalline polymers, copolymers, polymers in solution with low molar mass compounds, physical laminates or blends. The primary aim of using multicomponent systems is to mould the properties available from a single polymer to another set of desirable material properties. The property development process is complex and depends not only on the properties of the polymer(s) and other components but also on the formation process of the system which determines the developed microstructure, and component interaction after formation. Moreover, the process of polymer composite formation and the stability of the composite is a function of environmental parameters, e.g., temperature, presence of other species etc. The chemical composition and some insight into the microscopic structure of constituents in a polymer composite can be directly obtained using Infrared (IR) spectroscopy. In addition, a variety of instrumental and sampling configurations for spectroscopic measurements combine to make infrared spectroscopy a versatile characterization technique for the analysis of the formation processes of polymeric systems, their local structure and/or dynamics to relate to property development under different environmental conditions. In particular, Fourier transform infrared (FTIR) spectroscopy is a well-established technique to characterize polymers $[1,2]$.

While dispersive IR spectroscopic instruments have been less popular for long, most advances in the last thirty years were confined to FT systems due to their ease of use, small experimental times, high throughput, and high reproducibility. Obtaining FTIR spectra from polymeric samples is usually relatively rapid and straightforward. Moreover, the technique has attained high precision and accuracy in measurement to be sufficiently reproducible for most industrial, research and development purposes. Several experimental and post-data collection techniques for specialized applications not amenable to routine IR spectroscopic analysis have been developed over the years and a vast database of knowledge exists. However, IR spectroscopy had primarily been a "bulk" examination technique as obtaining spectral information from microscopic areas was difficult. The last fifteen-twenty years have seen considerable activity and development in the capability to collect IR spectra from small, specific regions of a sample. These developments have resulted in the development of a sub-field of considerable research activity - IR microspectroscopy. The set of techniques enabling microspectroscopic examination have demonstrated ability or great potential in examining the chem- 
ical structure to relate it to physical characteristics of materials and their fabricated products [3].

In this review, we present the current state of technology for infrared microscopic characterizations, illustrative applications, and possible future directions for this field with respect to characterizations of polymers. Infrared microspectroscopy, or the infrared spectroscopy of microscopic areas, can be broadly divided into three approaches based on the instrumentation used. The first class, using a large single element detector, is also the oldest. Such instrumentation has been commercially available for almost 15 years. The next approach, using array detectors, has been employed for a little over 5 years with commercial instrument availability at less than 5 years. Other approaches to microscopic detection are still being developed and are available in very few researchers' laboratories. FTIR microspectroscopy combines the spatial specificity of microscopy with the powerful chemical specificity of spectroscopy. Hence, any developed instrumentation or experiment is designed to emphasize some aspect of the spatial or chemical aspects of analysis. A balance between the need for sensitive spectral information essential for molecular characterization and the need for high spatial resolution visualization essential to morphological analysis is often achieved by instrumental and temporal limitations. Specific applications usually determine which of the spectral or the spatial characteristics are to be emphasized and what tradeoffs are tolerated. Hence, in this review, the three broad classes of infrared microspectroscopy are examined in sequence with emphasis on instrumentation capabilities and experimental possibilities. In analyzing applications, we focus primarily on the information-rich and well-characterized mid-infrared region of the spectrum, particularly suited for polymer analyses, and refer the reader to reviews on near-infrared applications [4].

\section{2}

\section{FTIR Microspectroscopy Using A Single Element Detector}

\section{1}

\section{Introduction}

While the first reports of a micro-capability spectrometer appeared 50 years ago [5], the technique made no major advances in applications to polymers until the coupling an interferometer and microscope to a digital computer enabled Fourier transform spectroscopy, time averaging, and mapping. The light throughput is used more effectively in the FT process and the development of more stable, sensitive, fast-response cryogenic detectors allowing for reproducible measurements further spurred interest in microspectroscopy. Infrared interferometers coupled to modern infrared microscopes incorporating sensitive detectors and a digital computer became commercially available in the 1980s and are, to date, popular analysis tools. There are estimated to be $\sim 5000$ systems currently in use. Spectral infor- 
mation from a small area of the sample can be obtained by restricting the area illuminated by the infrared beam using opaque apertures of pre-defined size. This allows microspectroscopic examination of small samples and can routinely be used to examine specimens down to the microgram range. However, single point examinations are of limited use and statistical viability in examining multicomponent polymers. By moving the sample in a known, pre-determined manner relative to the aperture, a point-by-point examination of a contiguous area of any size can be carried out. This technique is termed FTIR Mapping as by plotting the absorbance magnitude of a specific vibrational mode over the area, a map of that chemical species' relative concentration can be obtained. Maps of chemical species' abundance are also termed chemical maps or functional group maps. While optical microscopes use differences in refractive index, selective phase staining or polarized light for contrast, the spectral signature of a material provides the contrast in FTIR mapping. The non-invasive, non-destructive characteristics of IR spectroscopy are maintained while adding microscopic examination capabilities.

\section{2}

\section{Instrumentation}

Three major additions to the spectrometer are required for microspectroscopic mapping to be accomplished. First, the radiation from a modulated source has to be diverted to a microscope to focus on the desired sample area. A rapid scan interferometer is generally used as a source of radiation for SNR considerations, though the technique is not modulator specific. A step-scan interferometer, filter, or dispersive system may also be employed. No modifications are required to a standard interferometer and an attached computer can trigger interferometer scanning at a particular sample spatial position. Second, radiation reaching the detector is limited to be only from the area of interest on the sample. This is accomplished by using opaque masks or apertures, usually equipped with adjustable slit dimensions. Third, a positioning stage is required to precisely and reproducibly position the sample many times. Each stage movement is sought to be accomplished in as short a time period as possible. A computer is used to control the stage, interferometer, equipped to acquire optical images, and collect and store data. The IR microscope (shown schematically in Fig. 1) is very similar to the optical microscope. As there is no means to visualize the infrared image ab-initio, the sample has to be first visualized using optical methods. To obtain IR spectra from microscopic regions, apertures are employed first to selectively limit the wide field of view of the optical signal. IR radiation can then only pass through the slits and spectra are acquired by measuring this throughput as a function of interferometer retardation. The dependence of the infrared sampling on the optical image requires that the optical and infrared paths be parfocal and collinear. The major difference between optical microscopes and the one required for IR use is the incorporation of all-reflecting 

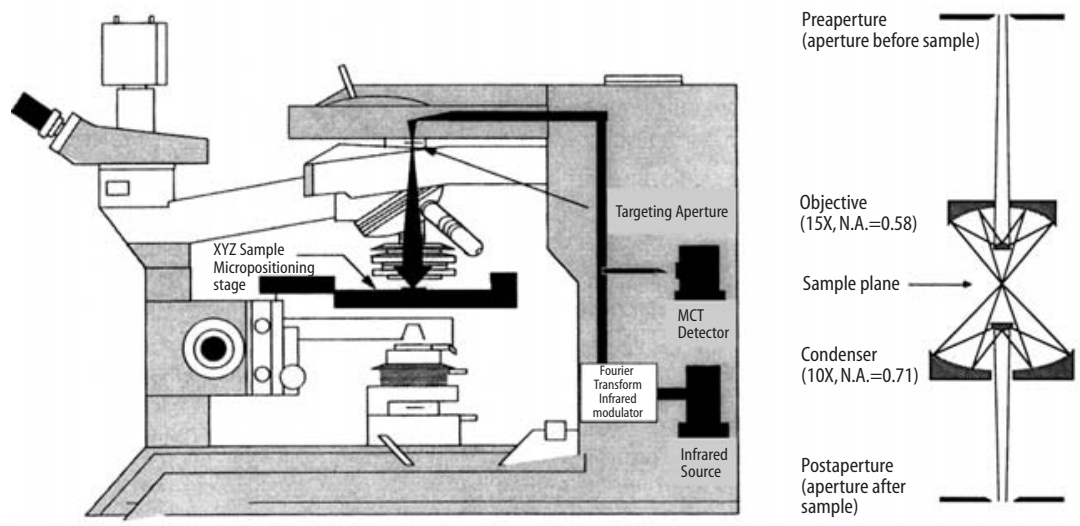

Fig. 1. Schematic diagram of an infrared microscope (left) reproduced from Koenig JL, Microspectroscopy of Polymers. The details of the focusing optics (right) are reproduced from Ref. [18]

optics to allow maximal IR transmission and the incorporation of aspherical reflecting surfaces in a Cassegrain-type configuration to minimize optical aberrations. Refractive elements, if required for some special needs, are made from IR transmitting materials that are resistant to moisture (e.g. $\mathrm{CaF}_{2}$ ). Apertures used are usually coated with highly absorbing carbon black to eliminate stray radiation. Polarizers or any other filters can be inserted into the beam path and polarized infrared measurements can be conducted similar to polarized measurements using visible radiation.

\subsection{1}

\section{Sampling Techniques}

Radiation incident on a sample results in radiation interacting with the sample to be transmitted, reflected, refracted, absorbed or emitted. Almost any of these modes [6] (Fig. 2) may be used for examining the absorbance characteristics in a microscopic configuration. However, microscopy places unique demands and some techniques have been more popular than others. Transmittance and reflectance techniques remain the most popular due to the ease of sample preparation and conduct of experiments. Light transmission through the sample allows for easy sample positioning, good light throughput and yields spectra that require little processing effort. However, sample preparation is more involved as most materials are strongly absorbing at their characteristic frequencies. The samples have to be thin, usually requiring experience and expertise in sample preparation in addition to the time and effort required to prepare optimal thickness samples. Microtoming a sample is often required and an appropriate embedding medium [7] may 

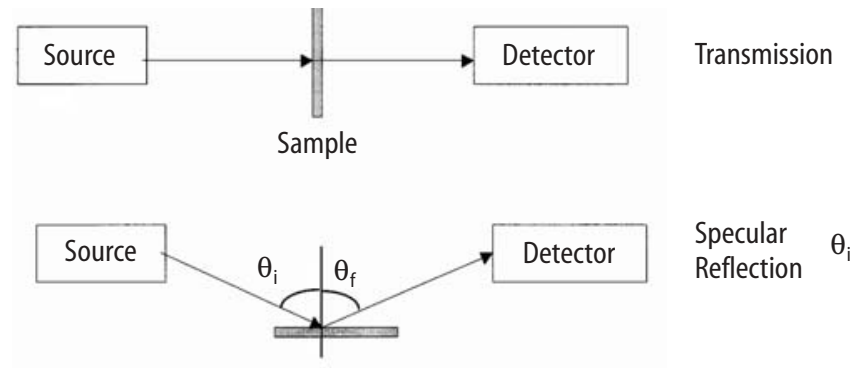

$$
\begin{aligned}
& \text { Specular } \\
& \text { Reflection }
\end{aligned} \theta_{\mathrm{i}}=\theta_{\mathrm{f}}
$$

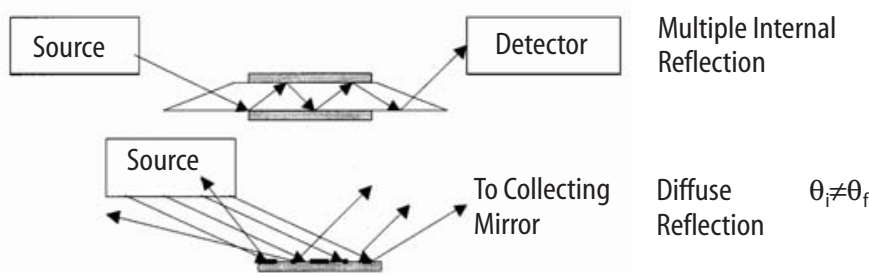

Fig. 2. Major Sampling Techniques for Infrared Spectra Collection in a Microscopic configuration

sometimes be used to handle a small sample before that step. While spectra may be obtained by flattening a sample [8] or using a diamond cell [9] to press the samples into thin layers, unwanted side effects of this approach may be manifest in terms of loss of orientation, interference fringes and distorted polarization. Once a good sample is obtained, quantification of data obtained from it is straightforward. The absorbance, $\mathrm{A}$, of a specific vibrational mode is related to the incident, $\mathrm{I}_{\mathrm{i}}$, and transmitted, $I_{t}$, intensities of radiation sample parameters as,

$$
A=a b c=\log _{10}\left(\frac{I_{i}}{I_{t}}\right)
$$

Where, $a$ is the absorption coefficient, $b$ the path length of radiation through the sample and $\mathrm{c}$ the concentration of the absorbing species.

Reflectance spectroscopy is commonplace for samples that cannot be prepared for transmittance measurements. However, reflectance measurements must be carefully conducted as the reflected beam is not only indicative of the composition of the sample but is also affected by surface conditions at the sample plane. This makes the reflectance spectra, though indicative of material chemistry, difficult to interpret and generally less useful for quantitative analysis. Since the polarization of the beam is maintained for reflectance, especially specular reflectance methods, examination of orientation at polymer surfaces using reflection techniques is attractive [10]. Reflection-absorption modes involve the transmission of the infrared beam through the sample and subsequent reflection to pass through the sample again. Usually, sample preparation is difficult for such experiments and they 
are useful when samples are thin in their native state and/or supported on a reflecting surface. Specialized accessories have been developed to control the angle of incidence and condense the beam after reflection. Using appropriate equipment, spectra from films as thin as a few nanometers can be observed. Attenuated total reflection (ATR) has been combined with the microscope to carry out ATR microspectroscopy $[11,12]$. An alternative approach has been developed that uses cartridges with hemispherical ATR elements and can be used in any microscope with reflectance capabilities [13]. Micro-Photoacoustic spectroscopy and time resolved microspectroscopy have also been reported. Adding to the versatility of the technique, temperature control may be achieved using a standard microscopy cell with IR transmitting windows [14]. There are no limitations to employing constant humidity and pressure (and vacuum) cells if the need arises. Special accessories [15, 16] can be readily incorporated by small changes in the optical configuration to examine different types of samples. FTIR microspectroscopy using polarized light provides information on the orientation of molecular species, which is especially useful for examining liquid-crystalline or semi-crystalline polymers and their composites. Infrared polarizers can be incorporated in microspectroscopic instrumentation to allow polarized radiation to impinge on the sample. Usually, two measurements, between which the polarizer is rotated $90^{\circ}$ are carried out to yield dichroic ratios of specific absorption modes. These can then be related to the relative orientation of polymer repeat units. However, the calculation of accurate dichroic ratios is predicated on the absence of stray radiation, polarization accuracy through the microscope optical train and focused optics at the sample. These conditions are difficult to maintain in a routine manner and thus, polarization experiments have not been widely carried out.

\subsection{2}

\section{Data Processing}

Data processing of mapped areas is mostly spectra based. Baseline correction of individual spectra, thickness corrections of individual area elements and other such plotting steps are routinely applied. The size of the data sets depends on the spatial resolution and area mapped. Given the large mapping time and low spatial resolution in most cases, the mapping technique is ideally suited to examining samples that have large spatial features, the discrimination of which does not require high resolution spectroscopy. Most often, the data sets are of a size smaller than $1 \mathrm{MB}$. Data processing is fast and many of the techniques applied to single spectra can be applied to extract information. Computation speeds and power requirements are moderate as only a few hundred spectra (at most) are ever processed. More complex data processing may be employed to improve the SNR, spatial resolution and component extraction. Surface contaminants as small as $10 \mu \mathrm{m}$ could be profiled by a data reduction program used to obtain projections about spatial locations. A 
method to co-add spectra from different positions was also suggested to improve spectral quality [17]. Since spectra collected are so few in number, it is usually more time effective to simply collect data for a longer time than apply any sophisticated techniques to improve the signal to noise ratio and extract information.

\subsection{3}

\section{Errors in FTIR Mapping}

The most glaring source of error comes from the component of the technique that facilitates microspectroscopy-apertures. Apertures are the largest source of diffraction in the IR microscope and can lead to the detector sampling light from outside the apertured region. The detector also samples the secondary lobes of the diffraction pattern. Thus, spectral information from the delineated area is spread over a larger area than the aperture. A case study revealed the effects of this stray light sampling as far as $40 \mu \mathrm{m}$ away from the sample (Fig. 3) [18]. Consequences of this stray light on spectra were studied under conditions of different aperturing modes and sizes. This problem can be circumvented by using a second aperture to delineate the same region (redundant aperturing). The spectral purity is now increased at a cost to the amount of light allowed through the system. Hence, spectral quality (SNR) is degraded. This requires very large collection times (loss in temporal resolution) or larger apertures (loss in spatial resolution). Thus, the three interdependent errors (stray light, low SNR and low spatial resolution) limit the effectiveness of the technique as a mapping tool to examine the spatial distribution of chemical species. Stray light not only compromises spatial fidelity, but may also lead to errors in determination of absorbance [19].

With dual aperturing in the mid-IR range, the practical resolution limit is close to $15 \mu \mathrm{m}$. This lower limit may be further increased to $\sim 10 \mu \mathrm{m}$, but at a substantial time cost. Typical collection time for a spectrum from a region is $\sim 30 \mathrm{~s}$. A further time of a few seconds is required to collect the data, process it, store it in the correct sequence, refresh data display and step to the next region before starting the next scan. Hence, to map a $500 \mu \mathrm{m} \times 500 \mu \mathrm{m}$ region with a resolution of $\sim 15 \mu \mathrm{m}$, it will take this mapping technique approximately 10 hours. Near field acquisition techniques have been suggested to improve spatial resolution and mask stray light. One such study [20] analyzed the acquisition of a physically masked sample (i.e. no mapping was possible). Another [21] proposed a near-field aperture turret to allow for mapping. Once again, the cost of improved resolution and accuracy is higher alignment and collection time.

Quantitative analyses require that spectra be measured from samples that are non-luminous, their absorbance be invariable with changes in concentration, the absorption coefficient of the species be independent of the intensity of incident radiation, the spectral response be uniform across the radiation cross-section, the instrument be devoid of stray light and respond in a linear manner to different lev- 

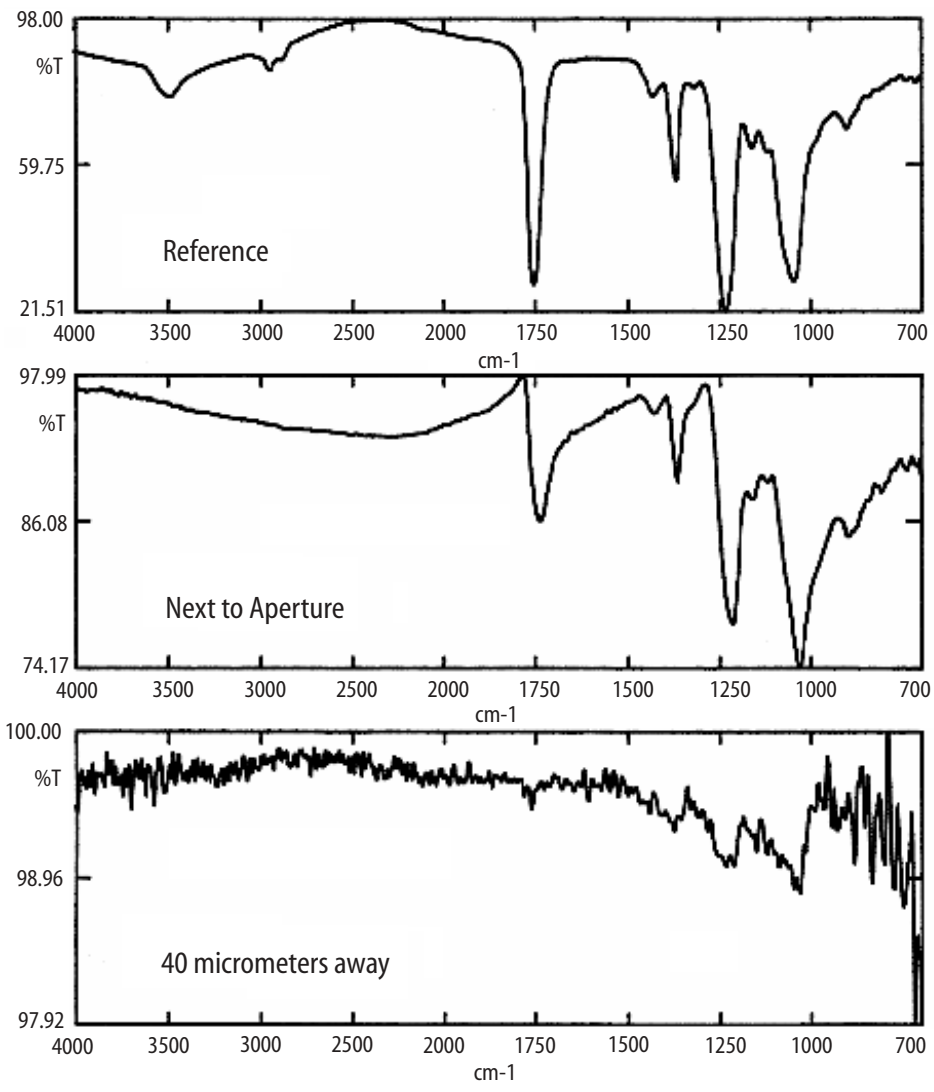

Fig. 3. The effects of aperturing can be seen on spectra collected with the aperture examining a polymer film (top) aligned with the film edge (middle) and $40 \mu \mathrm{m}$ away from the edge. (reproduced from Ref. [18])

els of light intensity. Some or many of the factors above are violated by sample non-uniformity, instrumental factors and the behavior of chemical species (interactions) leading to incorrect measurements. The effects of deviations can be theorized, quantified and experimentally verified. While diffraction has been found to be a major source of frustration, Siedel aberration may also serve to limit mapping fidelity [22]. In particular, centrally obscured reflecting optics (Fig. 1) are prone to spherical aberration effectively requiring adjustments to the optics after mapping small areas on the sample and limit the field of view for wide field analysis if the sample is not disturbed. In addition, the central obscuration reduces light throughput, further starving the detector of already scarce radiation. Numerical apertures of reflecting systems are typically limited (typically to less than 0.7 ) and magnification of no more than $50 \times$. This limitation is not important for routine FTIR microscopy in the mid-infrared and hence, reflective optics prove to be bet- 
ter suited - scientifically and economically - compared to refractive optics utilizing complex lens design. If the required wavelength range for infrared analysis allows for the use of materials (e.g. glass or $\mathrm{CaF}_{2}$ ) that may prove to be economically feasible, refractive optics that provide higher numerical apertures and spatial resolutions may be readily employed. However, chromatic aberration would have to be corrected for by appropriate microscope design [23]. Errors may also arise during use due to improper location of the spot to be mapped, substrate optical effects that shift the beam focus and loss of accuracy in system alignment over time.

\section{3}

\section{Applications}

A compilation [24] and discussion [25] of the applications of FTIR microspectroscopy to polymers is available. A concise introduction to the diverse applications can also be found [26]. Some newer and representative examples of various applications are presented below to illustrate the possibilities of IR microscopy. We have included more studies that seek to map an area rather than obtain spectra at discrete points. We believe the true utility of microspectroscopic instrumentation lies in examining a large area to find localized spectral changes akin to microscopy.

\subsection{1}

\section{Microsamples, Additives, Contaminants and Degradation}

Many polymeric samples are often available in small quantities for analysis. For example, new synthetic polymers are usually available in small quantities and forensic specimens may be limited in availability. In many situations, the identification and/or quantification of such samples are critical. However, samples smaller than the diameter of the probing beam lead to spectral artifacts, which complicate identification and quantification. Hence, many of these "microsamples" cannot be appropriately analyzed by conventional wide beam spectroscopy in their native state and microspectroscopy presents perhaps the only route to obtain artifact free spectra and consequently, the best chance of material identification.

Specially designed sample handling accessories can be used to localize the beam and/or control sample dimensions for obtaining a spectrum of the microsample. Hence, the sample does not need to be mixed with/embedded in a matrix. While small sized samples and concentrated contaminants can be detected, FTIR microspectroscopy can also be used to examine minute quantities of samples. While it has been claimed that concentrations in the nanogram range [27] can be detected, we believe that concentration levels down to $\sim 0.1 \%$ (w/w) of the volume of the beam at the sample can usually be quantified routinely. The sample preparation for such sensitive measurements is of crucial importance. Specifically, it is usually required that the material be isolated and pressed into a thin film over a spot size 
of $\sim 15 \mu \mathrm{m}$ to obtain the best spectra. Many specialized sample handling accessories are available and can be employed to accomplish this task or provide alternate sampling geometries if pressing the sample into a film is not desirable or viable. One such microsampling accessory, the Diamond Anvil Cell (DAC), allows for small sample handling and applies pressure to the sample, which can be employed to change the dimensions of the sample.

The sensitivity of FTIR spectroscopy to microscopic defects is greatly increased compared to a macroscopic measurement - if the defective area is examined. As opposed to single element, wide beam spectroscopy where the spectrum from the entire sample is obtained, the polymer does not overshadow the contaminant if a spectrum from a microscopic area of the sample is obtained. The contaminant can be later identified from a library of known compounds, providing clues to process control and helping to maintain quality control. This approach has been used to identify gel inclusions in poly(ethylene) $[28,29]$, contaminant on the surface of a semi-conductor device [30], acrylic fiber on a microcircuit die (Fig. 4) [31], contaminants in poly(vinyl chloride) (PVC) [32] and a mold release agent on the surface of a polyurethane [33]. An additive (erucamide) in linear low density polyethylene (LLDPE) that was found to migrate to the surface [34] thereby changing the surface properties could be readily identified. Various contaminants, whether in a
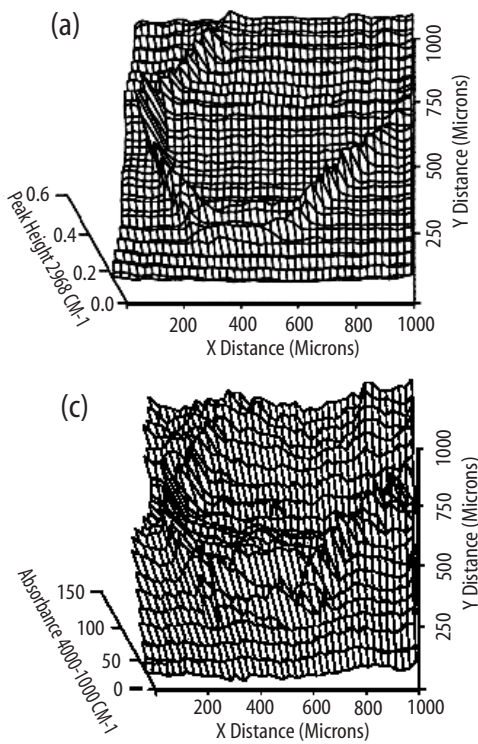
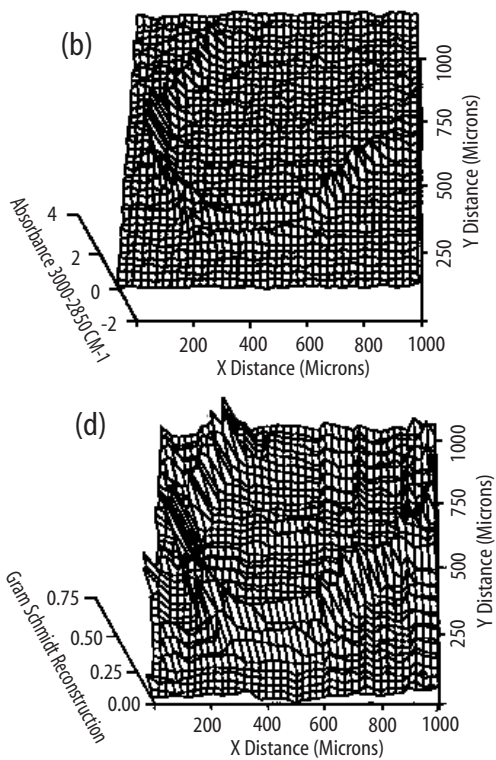

Fig. 4. The distribution of a contaminant is examined by reconstructing images of an acrylic fiber contaminant on a microcircuit die using various methods of visualization. Image generation methods include the use of $\mathbf{a}$ absorbance intensity at $2968 \mathrm{~cm}^{-1}, \mathbf{b}$ integrated absorbance between 2850 and $3000 \mathrm{~cm}^{-1}$, c integrated absorbance from 1000 to $4000 \mathrm{~cm}^{-1}$, and $\mathbf{d}$ the Gram-Schmidt method. (reproduced from Ref. [31]) 
film on the surface, as inclusions in the bulk or simply attached to a polymer device can be identified using appropriate techniques to obtain a sample with the contaminant. In a few minutes of experimental time, the presence and identity of the contaminant can be readily determined.

Contaminants may be identified by two approaches: optical microscopy is first used to identify an anomaly followed by its isolation in the IR field of view by apertures. A spectrum is then acquired from the small, delineated area to identify the contaminant. Clearly, the identification of the small area containing the suspected contaminant using light microscopy is critical. Optical contrast needs to be sufficiently high to allow such delineation and the question as to the contaminant's identity can be resolved by microspectroscopy. Alternately, the whole sample area is mapped and a contaminant can be identified if an anomalous signal is observed from a small area in the map. Mapping is especially useful when the optical detection of contamination is difficult due to the heterogeneous nature of the sample or little difference in refractive indices between the sample and the contaminant. General mapping of sample areas has also been known to lead to serendipitous discoveries of contamination or to the discovery of more than one type of contaminant/degradation product. For example, the degradation of poly(propylene) (PP) was studied using FTIR mapping [35] indicating the presence of many different types of degradation products. Similarly, localized degradation photoxidation of polymers [36] can also be examined whether on the surface or in the bulk after microtoming. Other case studies reported have included lubricant in nylon [37], pyrolysis products from microsamples, inclusions in PVC, inclusions in a rubber sample and growth of foreign material in a brewery pipe [38]. Process contaminations can be readily examined, for example, anomalous material on a magnetic disk during manufacture was found to be cellulosic with some kaolin clay, probably from a coated paper used in the manufacturing process [29]. Another contaminant reported in the study was similar in visual appearance; but was found to be a polyamide that matched the Nylon resin used. Yet another contaminant on the disks, which could not be distinguished by optical microscopy alone, was found to be a polyglycol. Thus, different residues due to manufacturing defects can be readily identified and the process step where the contamination occurred can be monitored and/or modified.

The degradation of a polymeric sample often begins at the surface and proceeds into the bulk. Injection molded samples of polyamide 6,6 [39] were aged at elevated temperatures and examined to determine changes. Skin-core oxidation was observed and evaluated by infrared spectroscopy and by imaging chemiluminescence. The oxidation depth profiles determined by the two techniques using the carbonyl stretching frequency (FTIR) and the peroxide depth profiles (chemiluminescence) showed good agreement and the results indicated a diffusion-controlled oxidation process. Thus, not only can a material be monitored for contamination and/or degradation but also, in some cases, the mechanism and the source of the 
anomaly can be probed to allow characterization of the process which lead to the appearance of the anomaly or failure of the product.

\subsection{2}

\section{Single Polymer Fibers}

Single component, multicomponent fibers [40] or fibers with dyes [41] or pigments [42] that have diameters down to $10 \mu \mathrm{m}$ can be detected and analyzed by FTIR microspectroscopy. Cotton, wool and acrylic fibers could be readily differentiated by examining their infrared spectra [43]. Fiber blends (cotton/terry vs. cotton/polyester) could also be differentiated. PET fibers $[46,44]$ and a polysulfone fiber in an epoxy matrix could be characterized. Groups of fibers classified according to size generally require different methods of sample preparation [44]. Fibers larger than $\sim 30 \mu \mathrm{m}$ in diameter did not require much sample preparation. For smaller fibers, better results were obtained by effectively increasing the sample width by flattening the sample using a DAC. ATR microspectroscopy can also be employed to provide information when transmission experiments are difficult or surface weighted information is required [45].

While the detection and identification of single fibers can be readily accomplished using microspectroscopy, the quantitative analysis of orientation is complex [46]. Polarized intensities obtained from a large film using microscopy and conventional wide beam illumination were found to agree well. However, a lensing effect similar to that of using variable path length cells [47] due to the curvature of fibers is observed when fibers are examined. This lensing effect complicates identification and severely affects the determination of orientation. To eliminate the lensing effect, flattening the fiber is suggested to make the surfaces parallel and the specimen larger. However, this process may result in loss of the original orientation in the fiber. The effects of polarization scrambling by the system optics, diffraction effects and stray light can be reduced by proper system design to yield better quantification of orientation. The use of physical masking at the sample plane was shown to result in high photometric reproducibility. By applying many of these experimental protocols, high quality spectra and structural information can be obtained reliably for many fibers [48].

\subsection{3}

\section{Polymers in Biological Areas}

Polymers are used for biological applications in a variety of situations from drug carriers to components of artificial limbs. In an instance of replacement of live tissue, PTFE grafts are used to treat lower-extremity ischaemia when autologus saphenous veins are unavailable. As a step toward understanding the ensuing complications and tissue build-up, a graft was simulated in a flow device [49]. The con- 
centration of lipids within the grafts was monitored by FTIR microspectroscopy. Lipid uptake was found to be rapid initially and then slowed down over time. These model studies are important in developing an understanding of the transport in porous vascular prosthesis and FTIR spectroscopy and microscopy present a sensitive analysis tool to detect composition changes in biomaterials.

The oxidation and subsequent degradation of polymers may lead to a loss of mechanical properties and product failure for biological devices. One area where performance is critical and is affected strongly by polymer degradation is the use of implanted prosthetic devices. Ten new polyethylene prosthetic components (PEs), sterilized by two different routes were examined using FTIR-ATR and microscopy [50]. All the samples showed some degradation due to the formation process. However, by microscopic measurements and derivatization techniques, the ethylene oxide (EO)-sterilized prosthetic components were shown to be different from the gamma-radiation sterilized. EO sterilized devices had low levels of depth oxidation similar to degradation during preparation of samples. Gammasterilized devices showed higher oxidation levels, variable from sample to sample, on the surface and in the bulk. The oxidation is described both by carbonyl species distribution and by hydroperoxide concentration. Not only could differences in degradation but the scheme of degradation could also be inferred by IR analysis. Chain scission of gamma-radiated PE, estimated by IR analysis, results in reduction of molecular weight and lowers its abrasion resistance. Thus, the effects of the sterilization methods could be observed. The example illustrates the applicability of FTIR microspectroscopy in product development where FTIR microspectroscopy is especially attractive given the relatively low cost of instrumentation and the large amount of information provided in most instances. In a similar vein, the effects of near-colon activity on a cross-linked polymer were examined in-vitro [51]. A combination of conditions of use and rapid and accurate analysis is a cornerstone of the development process and FTIR microspectroscopy can often play a role.

\subsection{4}

\section{Semi-Crystalline Polymers}

Poly(vinylidene fluoride) is an interesting polymer that has a large number of crystal phases, which determine its performance properties. The crystal structure of PVDF has been analyzed to identify its various phases [52]. The different types of spherulites seen optically were assigned to different crystal modifications using FTIR identification. Blends of PVDF with PMMA were analyzed to determine differences in interaction based on PMMA tacticity [53]. On the basis of shifts in the carbonyl region, it was suggested that i-PMMA had stronger interactions with PVDF than s-PMMA. The crystalline and amorphous regions of another polymer with potentially wide application, Nafion [54], were examined by FTIR microscopy. The performance of the fuel cell is critically dependent on the water uptake in 
the polymer electrolyte. In the crystalline regions, sulfonic acid groups are present at the end of the side chains. Thus, the crystalline regions contain no water molecules. In the amorphous regions there is a complete proton transfer from the acid to the water molecules. As a result, sulfonate groups are obtained and water retention is facilitated. Such microstructure determinations, which affect performance properties and are critical for quality control, can be routinely carried out using FTIR microscopy.

External nucleating agents often facilitate polymer crystallization and a variety of analytical techniques can be employed to monitor the effects of these agents. The effect of seeding agents (saccharin, phthalimide and boron nitride (BN)) on the rate of crystallization of polyhydroxybutyrate (PHB), and its blends with poly (hydroxybutyrate-co-valerate) (CHB/HV) [55] was investigated using optical microscopy and DSC. However, most of these techniques are incapable of providing the distribution of chemical species across the various morphological features while the chemical constitution in separate local areas can be readily determined by IR microspectroscopy. It is well known that the interaction of the nucleating agent with the (crystallizing) polymer can determine the effectiveness of the crystallization method. Thus, in the study described above, the solubility of the nucleating agent in the molten polymer was determined. Saccharin and phthalimide were found to be poorer nucleating agents compared to $\mathrm{BN}$. They were found to be soluble in molten PHB, but were rejected from the crystalline PHB. On the other hand, small crystallites of saccharin and phthalimide developed within the boundaries of the spherulite, but most material accumulated in the inter-spherulitic domains. Thus, not only the interaction but also morphological location of nucleating agents can be determined. Just as crystal structure heterogeneity can be detected, the homogeneity can also be determined. Thin Polyimide (PI) films formed by vapor co-deposition of the precursor molecules pyromellitic dianhydride(PMDA) and 4,4'-oxydianiline(ODA) were examined for spatial homogeneity [56]. No evidence for PDMA phase separation or crystallization variation was found. FTIR microspectroscopy, combined with polarized radiation from the interferometer, is a powerful means of simultaneously determining local composition and relative organization in crystalline polymers often providing a complementary tool to X-ray scattering [57], DSC [58] or microscopy studies [52, 59].

\subsection{5}

\section{Phase Separated Polymer Blends}

Phase separated blends of poly(vinyl alcohol) [PVA] and poly(vinyl acetate) [PVAc] were studied and evidence of intra-molecular and inter-molecular specific interactions was reported to depend on blend composition [59]. Blends with high PVA content were reported to have large intra-molecular specific interactions while blends with a high PVAc content showed inter-molecular interactions. The 
morphology was characterized by Fluorescence optical microscopy (FOM). PVArich domains were identified by green fluorescence of fluorescein while PVAc-rich domains were identified using the blue fluorescence of anthracene. The two polymers did not require any staining or tags to be distinguished by infrared spectroscopy. However, the FTIR mapping spatial resolution is often insufficient to determine morphological information like size and shape requiring additional microscopic approaches. A model polymer blend system [60], poly(methyl methacrylate) [PMMA] and poly(styrene-co-acrylonitrile) [PSAN], was studied in an attempt to characterize the phase diagram of the blend system. Poor correlation was found between the phase diagram determined in this manner and the phase diagram obtained by optical microscopy. The inconsistency probably arises from the large spot size of the infrared beam and the lack of spatial fidelity of apertured spectra. Olefinic blends are often used in utility polymeric articles. In a model blend that was studied, the refractive indices of the two components- isotactic PP and syndiotactic PP- were close enough for their phase separated blend to be deemed single phase by optical microscopy. However, chemical mapping of the sample [52] shows clear phase separation based on spectral differences (see Fig. 5).
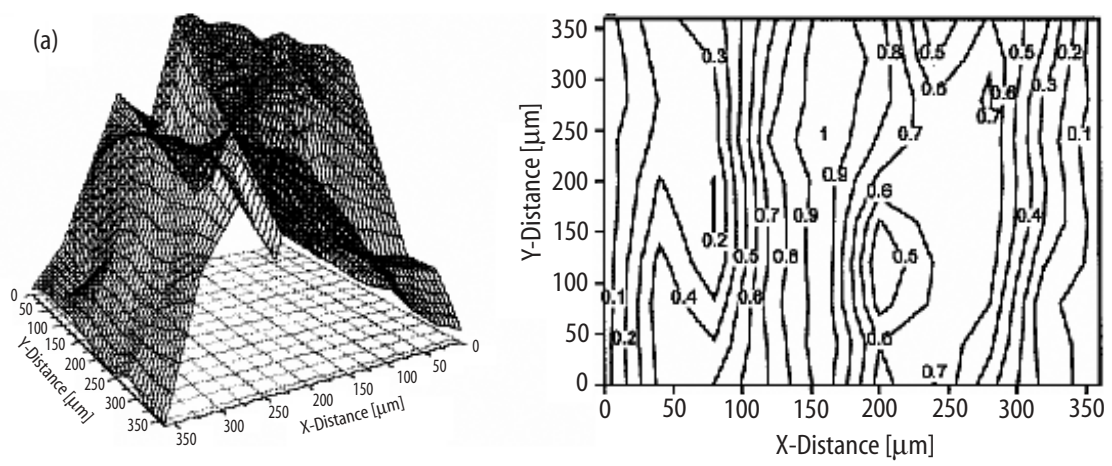

(b)

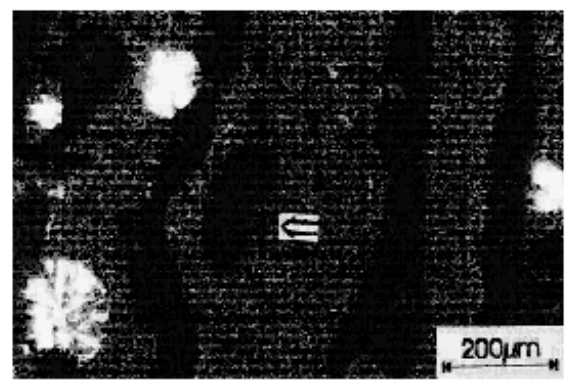

Fig. 5. The functional group image $\mathbf{a}$ of a region in a polymer blend of i-PP and s-PP. A contour plot is another visualization useful for the same. b The optical photograph reveals that the dark portion is s-PP surrounded by i-PP (Ref. [52]) 
These examples also illustrate the difference in spatial resolution and contrast mechanisms between optical and infrared microscopies. While optical microscopy is capable of higher spatial resolution, its discrimination is limited to a difference in the average of a property of materials, namely refractive index, unless specially labeled to detect a property of the label. Infrared microspectroscopy derives its contrast mechanism from the intrinsic composition of the material but suffers from a poorer spatial resolution. A judicious use of the two complementary techniques is often required to achieve good characterization. While the example above illustrated the detection of differences, FTIR microspectroscopy can also be used to determine homogeneity. For example, compositional differences in a PP-PE film could not be detected between the surface and up to $500 \mu \mathrm{m}$ into the bulk of the sample [61].

\subsection{6}

\section{Multilayered Polymer Systems}

Multilayered polymer films are increasing used in packaging to take advantage of the properties of individual polymers. Often, the two (or more) polymers used are incompatible and a tie layer of another polymeric adhesive has to be used between them. This leads to complicated laminate systems with more layers than required for simple barrier properties. The analysis of such films has been reported many times in the literature [37,62]. A laminate is usually microtomed along the thickness direction and mapped. A microtomed laminate has often been a popular one to compare microscopy techniques with other FTIR or microscopic techniques. In one such early comparison using a laminate [63], it was shown that the spectra obtained by microscopic analysis were much superior to the ones obtained by an ATR-FTIR technique. Additionally, microtoming allowed for examination of an additional dimension, where sections taken progressively in one direction could be sequentially compared. Poly(urethane) coated on an ethyleneacrylic acid (EAA) (15\% acrylic acid) was studied in this manner to find that the polyurethane had penetrated $60 \mu \mathrm{m}$ into the EAA layer [64]. Dimensions in the tens of microns are directly accessible by FTIR microscopy. For dimensions smaller than tens of microns, spectral subtraction may yield qualitative information about the identity of a middle layer, the location of which has to be identified optically. Mathematical analysis methods have also been developed for detection of such layers. A factor analysis method can be used in cases where the thickness of the layer is smaller than the resolution of the microscope [65]. The resolution of a 2-3 $\mu \mathrm{m}$ thick inner layer, from a four-layer polymer laminate was achieved by self-modeling multivariate analysis [66]. Quality control of packaging materials, the performance of which may be critical to the packed materials, can be routinely carried out to verify local microscopic structure, detect defects and contaminants. 


\subsection{7}

\section{Diffusion}

The approach of forming an interface by laminating films of two polymers, allowing them to diffuse and then microtoming a vertical section to obtain a sample for IR mapping is a popular one to examine diffusion. Spectral profiles along the diffusion direction can be obtained and the concentration of the diffusing species directly inferred. This procedure has been used to analyze the interdiffusion of poly(acrylic acid) (PAA) and poly(ethylene glycol) (PEG) [21]. The concentration profile was found to be consistent with Fickian diffusion. The interdiffusion of poly(ethylene-co-methacrylic acid) [EMAA] and PVME [67] could also be studied in a similar manner. The diffusion process also affects the adhesion process strongly. Effects of the diffusing species' (PEG) molecular weight and contact time on diffusion across the interface between two hydrogels were investigated [68]. Results indicated that diffusion enhanced the adhesion between the hydrogel layers. However, the study of diffusion is often hampered by the limited spatial resolution afforded by FTIR mapping systems and the large times required to map the diffusion gradients. Thus, examinations of diffusion have been limited to fast polymer or oligomer pair diffusions. Polymer dissolution by small molecular weight solvents has been too rapid to examine using mapping techniques and the diffusion profiles for very limited low molecular mass substances have been mapped. The diffusion of an anti-oxidant into PE was one of the first reported examples [69]. The diffusion Cryasorb UV531 in PP [70], and Bovine Serum Albumin (BSA) in amylopectin [71] has also been examined. Diffusion of olive oil into polypropylene was investigated and found to be Fickian with constant diffusivity [72].

The processes of reaction and diffusion occur at the same time in a variety of systems. These issues are particularly important in the formation of blend systems and are central issues in the performance property enhancement of such systems. A study of the competitive effects of the rates of the two processes can be easily carried out using FTIR microspectroscopy. The rate of diffusion can be monitored by the time evolution of the absorbance (concentration) profiles while the rate of reaction can be monitored as a time evolution of the reactant (or product) absorbance (concentration). Reaction of a random copolymer of styrene and maleic anhydride (SMA) with bis(amine)-terminated poly(tetrahydrofuran) (PTHF) is one such studied system [73]. Temperature was varied while studying the effects of two different PTHF molecular weights. The reaction rate constants were obtained from the initial slope of conversion-time plots. In addition, it was shown that the rate of diffusion was faster as diffusion of PTHF into the SMA phase occurred prior to the imide formation. The imide was formed in the SMA phase and quantitatively estimated. A corresponding decrease in the carbonyl stretching vibration of the maleic anhydride peak was seen. 


\subsection{8}

\section{Local Orientation}

4,4-diaminodiphenyl methane (MDA) and 4,4-diphenyl methane diisocyanate (MDI) were polymerized using vapor deposition to form poly(urea). The dipole orientation of $\mathrm{NH}$ and $\mathrm{CO}$ dipolar groups caused by corona poling was analyzed. In diode corona poling, the dipoles are aligned greatest in the area directly below the needle electrode while in triode corona poling, a grid electrode is set up between the needle electrode and planar electrode. Hence, it is expected that orientation is accomplished uniformly over the entire surface. However, using infrared reflectance microspectroscopy, dipole orientation was found to follow the form of a net mesh structured like a grid electrode [74]. Orientation due to other formation processes, for example in extrusion molding, can be analyzed. Sheets of a thermotropic liquid crystalline polymer, consisting of 4-hydroxybenzoic acid, phenol and dicarboxylic acid units were examined [75]. The (relative) orientation of molecules and sub-molecular species is important for the properties of many novel optical and electrically responsive devices. Local structure of polymeric materials can usually be analyzed in the polymers native state or on the device itself. Given that devices are becoming smaller, the use of microspectroscopy to determine local structure can be expected to become more relevant.

The local orientation in polymers can also be examined in the context of polymer failure. Two types of polyisoprene samples were stretched until cracking was initiated and the crack tip was subsequently examined using FTIR mapping [76]. Unfilled polyisoprene samples revealed a uniform infrared spectrum for regions surrounding the crack tip at the resolution of the FTIR microscope. Silica filled samples, however, had demonstrated enhanced absorbance levels of the $\mathrm{C}=\mathrm{C}$ stretching frequency ahead of the crack tip, the region corresponding to the highest stress. Preferential orientation was also observed ahead of the crack tip by employing dichroic ratio measurements. Hence, the molecular changes induced by stress close to the failure region could be observed by combining the sub-molecular sensitivity of FTIR spectroscopic measurements with the spatial discrimination afforded by the microscope accessory.

The local orientation distribution in polymeric samples due to self-organization (e.g., liquid crystalline polymers) or due to processing-induced orientation can be examined using polarized measurements. A relative measure of the distribution of orientation in a sample, such as comparison of surface orientation to orientation in the polymer bulk [77], can be accomplished by polarized microspectroscopy. An examination of extrusion-molded sheets of a liquid-crystalline copolyester [77] revealed higher surface orientation compared to the bulk. The orientation function can then be related to processing conditions, for example drawdown ratios [78]. Since the draw-down ratio can be related to physical properties, a measure of the orientation function provides a route to relate the microscopic 
chemical organization to macroscopic properties. By suitably changing the processing conditions, the spatial differences in orientation can be decreased and processing conditions for making polymers with uniform physical properties can be determined.

\subsection{9}

\section{Polymer Surfaces and Interfaces}

By their very nature, surfaces are usually heterogeneous. Sometimes, the structure or changes in structure across a surface or at the interface of two surfaces is important in determining the properties of the polymer composite. Usually, IR spectral surface analysis can be carried out using non-spatially resolved IR spectroscopy. Analyses of surfaces (with respect to depth below the surface) can be carried out by microtoming the sample. Similarly, microtoming is a useful technique for polymer interfaces and polymer-metal interfaces if the metal is present as a thin film. Reflection techniques can also be used to obtain spectra from samples without microtoming. A spatially resolved method was used to determine effects of different surface treatments on the same epoxy film [79] and chemical effects of water ingress [80]. $-\mathrm{SO}_{2} \mathrm{OH}$ clusters at the surface and embedded below the surface were observed as a function of environmental conditions for Nafion-H, silica-supported Nafion-H and two types of Nafion-H silica nanocomposites [81]. Specific interactions were observed, probably arising from the interface, for silica systems as evident by shifting and splitting of the OH peaks. Biodegradation of thin LDPE film in soil was studied and found to occur relatively fast [82]. This was attributed to the synergistic action of oxidative and/or photo-oxidative degradation on biological activity attributed to the increasing hydrophilicity of the film surface.

\subsubsection{0}

\section{Polymer-Liquid Crystal Systems}

At this point, it is instructive to examine the contact method (Fig. 6). When a solvent is brought into contact with a polymer film, it diffuses into the polymer. If the film is constrained between two IR transparent substrates and the liquid is forced into the gap by capillary action, the contact and subsequent diffusion of the liquid can be easily monitored by means of chemical imaging using light transmitted in the perpendicular direction. After contact, diffusion between the two materials starts. The absorbance (concentration) profile can be measured with accuracy to yield diffusion profiles [83]. However, for liquids, where capillary action is facilitated, the examination of concentration profiles in-situ is difficult. If the concentration profiles developed at higher temperatures are examined at a temperature that prevents further diffusion, the profiles are frozen-in. The diffusion of E7 into poly(butyl methacrylate) (PBMA) was studied [84] in this manner and compo- 


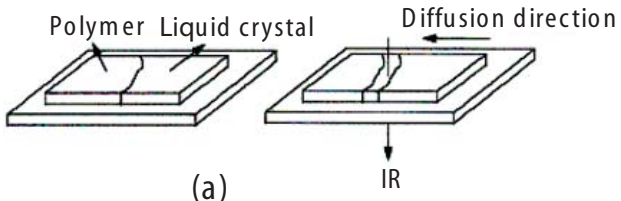

(a)

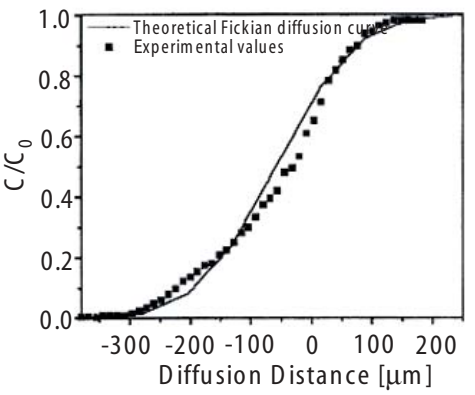

(c)

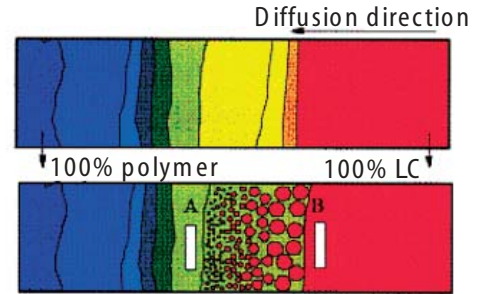

(b)

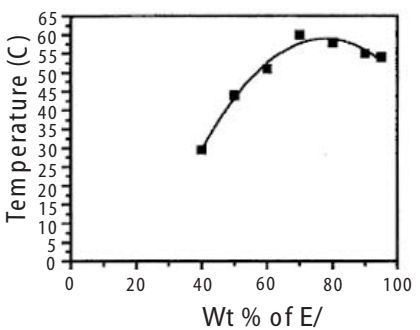

(d)

Fig. 6. The contact method (a) involves the introduction of two soon-to-be diffusing materials between substrates. Capillary action brings the liquid and solid in contact. This sets up an area of varying concentration due to diffusion (b) top. Upon Polymerization or cooling, this some part of this region may phase separate (b) bottom. The apertures seen in (b) can be sequentially moved to extract diffusion profiles (c) or phase diagrams (d) (from Ref. [84])

nents of E7 were shown to diffuse at different rates [85]. The component partitioning could be detected based on small spectral differences between the four components monitored as a function of time.

The contact method in conjunction with FTIR microspectroscopy has also been used to determine the phase diagram of an LC-cured pre-polymer pair. An LC mixture (E7) and pre-polymer (NOA65) were allowed to diffuse for some time to achieve a concentration gradient. The pre-polymer was subsequently polymerized by UV initiation. Under the optical microscope of the spectrometer, phase separation was observed in certain regions. The regions on either side of the phase-separated band are single phase and the highest concentrations in these regions, determined by microscopic FTIR measurements, yields the solubility limit. Repeating this procedure over different temperatures gave the phase diagram [86]. PDLCs were characterized to obtain spectra from the droplet and matrix regions as well as examine local LC orientation (see Fig. 7) [87]. The influence of the matrix material on local order and resulting droplet configuration could also be identified [88]. The time resolved response of microscopic regions in large and small PDLC droplets could also be obtained [89]. 
A
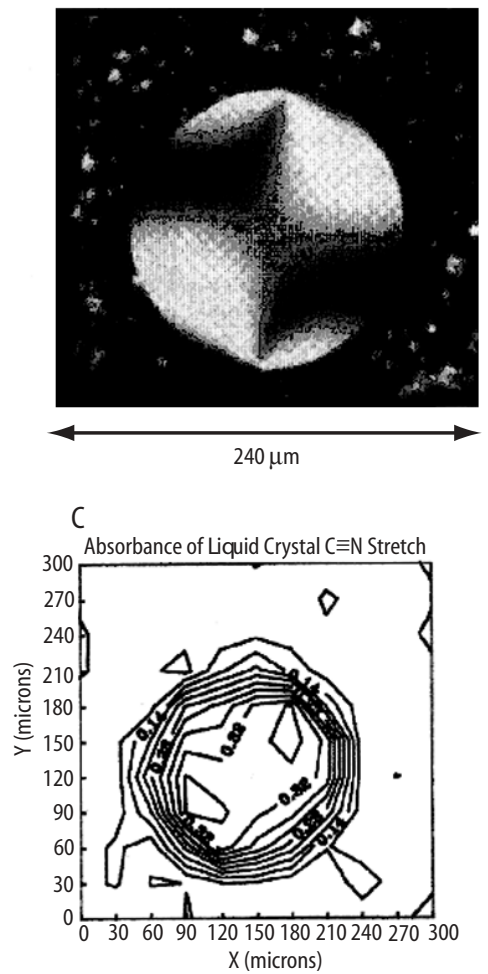

B

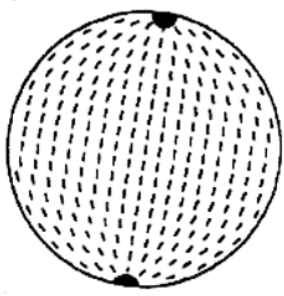

D

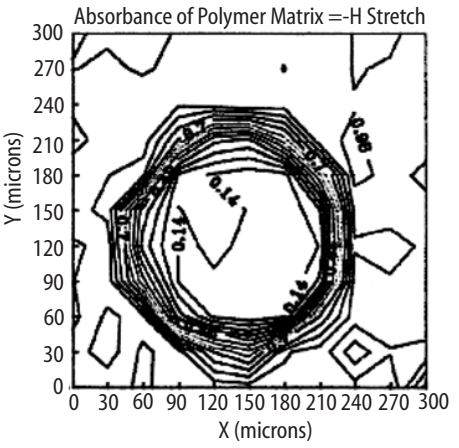

Fig. 7. Infrared Contour Maps of a liquid crystal specific peak (left, bottom) and a matrix (epoxy) specific peak (right, bottom) of a PDLC droplet, whose polarized optical image is shown (top, left) with the corresponding orientation visualized (top, right) (from Ref. [87])

\subsubsection{1}

\section{Filled Systems}

\subsubsection{1}

Carbon Black, Silica and Inorganics Filled Systems

Two major fillers used in the rubber industry are silica and carbon black. Carbon black is black because it absorbs/scatters all radiation, including infrared, impinging on it. Hence, simple transmission spectroscopy of carbon black filled specimens is not straightforward and is usually not possible unless the samples are very thin. Carbon black filled samples have not been readily examinable using microspectroscopic methods. Silica filled systems are more amenable to microscopic techniques [76] and can be examined to determine silica-polymer(rubber) interactions. The presence of inorganic materials, e.g., transition metal complexes in 
polymers, can be examined by FTIR mapping. In one such study, the distribution of the metal complex was found to be homogeneous [90], thus validating the formation procedure.

\subsubsection{2}

\section{Fibers and Fillers}

Fiber reinforced epoxies can be used to obtain materials with good mechanical properties. However, a point of failure is the poor adhesion between the fibers and epoxy. Hence, surface adhesion was sought to be improved using surface modification techniques and analyzed using FTIR mapping experiments. The fiber-matrix interphase was examined for chemical reactions in Kevlar-epoxy composites [91]. The amine groups in Kevlar were shown to accelerate the curing process (see Fig. 8). The interphase of as-received and modified glass fiber-epoxies could be obtained by least squares and difference analysis of thickness corrected maps [92]. Environmental ageing effects due to humidity on these composites were simulated and the composites mapped to find preferential water accumulation close to the fiber-matrix interphase [93]. Using a silane coupling agent decreased the effects [94]. The effects of environmental conditions on fibers themselves can be readily examined using FTIR microspectroscopy [95]. Simultaneous reaction and orien-

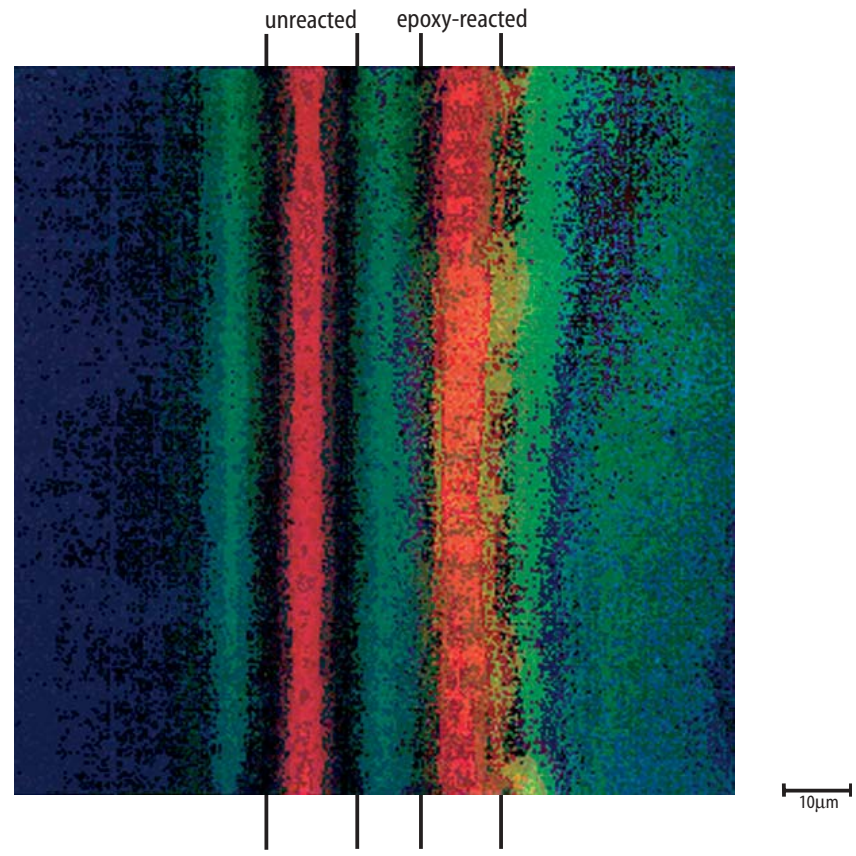

Fig. 8. FTIR maps of the reacted and unreacted fibers from the NH stretching vibration at $3326 \mathrm{~cm}^{-1}$ (from Ref. [91]) 
tation information could be obtained in a case where nitrile reaction, conjugated $\mathrm{C}=\mathrm{C}$ formation and oxidation occurred at the same time to possibly affect orientation.

\section{4}

\section{Summary}

The single detector element microspectrometer was demonstrated over 50 years ago. Systems today employ FTIR spectrometers, sensitive and stable detectors and high-speed computers with large storage capability, which allows for the mapping of millimeter size spatial areas. However, due to the sequential nature of the mapping process, the experimental time is large. Hence, for spatial distribution information of chemical species, FTIR imaging is becoming popular (vide infra). However, the single detector element systems are not very expensive and remain the method of choice when discrete measurements from a number of points have to be made, experimental time is small compared to the time scale of changes in the sample, high spatial resolution is not important, or a spectrum from a small sample is required. These qualities have made the single element microscopy spectrometer a useful tool for industrial applications while providing unprecedented information for research activities.

\section{3}

\section{FTIR Imaging}

\section{1}

\section{Introduction}

The state of the art in FTIR microspectroscopic instrumentation today is the combination of a Focal Plane Array (FPA) detector and a step-scan interferometer [96, 97]. FPA detectors consist of thousands of individual detectors laid out in a grid pattern. Hence, the interferometer signal is simultaneously sampled by each detector leading to a multiplexed spectral and multichannel detection advantage. Spectral discrimination is provided by the interferometer and spatial resolution by discrete detector elements on the FPA. Each FPA element corresponds to a specific spatial region on the sample and no apertures are required to restrict radiation. A schematic of the setup may be seen in Fig. 9. This configuration allows for spatially resolved imaging of a wide field of view in a data collection time a little more than for a single pixel. From the results of the imaging experiment, a spectrum from a small region may be examined or spectroscopic signal from a spectral feature plotted for the field of view, providing simultaneous examination along spatial and spectral dimensions. This technique considerably reduces experimental time for imaging static samples and has enabled the examination of many dynamic processes [98]. 


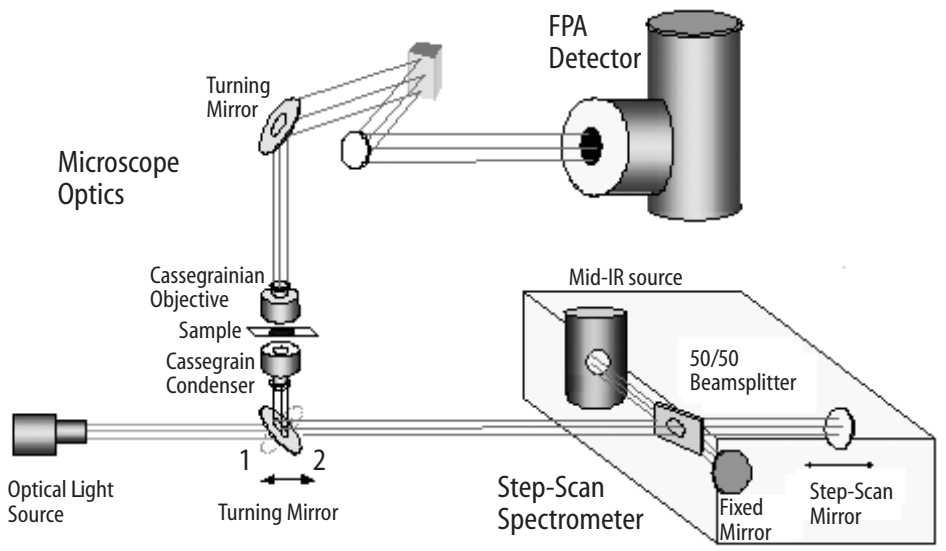

Fig. 9. Schematic Diagram of an FTIR Imaging Spectrometer

\subsection{1}

\section{Mapping Versus Imaging}

A comparison of the mapping and imaging techniques has been carried out [99]. As explained above, the sample is imaged onto the FPA using the magnification of auxiliary optics such as a microscope. The optics determine magnification and hence, the nominal spatial resolution afforded by the size of the individual detectors in the array. No apertures are required to limit the sample area examined in an imaging experiment and the sample does not need to be moved as a given field of view is imaged in a single collection experiment. It may be immediately seen that the collection time is decreased by a factor of $n^{2}$, where $n$ is the number of spatial resolution elements in one direction of a square sample area imaged. Analogously, $n$ is the number of steps in a mapping experiment. Neglecting the effects of resolution, a mapping step may be considered equivalent to a pixel in the imaging experiment. However, the practical spatial resolution limit in the mapping technique is usually $\sim 15 \mu \mathrm{m} \times 15 \mu \mathrm{m}$ and additional time is required to move the sample from position to position. For imaging, the resolution is essentially wavelength limited but additional time may be required to process the large data sets that result.

As an example to compare the two techniques, a $64 \times 64$ element array imaging a $500 \mu \mathrm{m} \times 500 \mu \mathrm{m}$ area will provide reasonable quality spectral information in less than a minute. The same measurement at a resolution of $\sim 15 \mu \mathrm{m}$ will take approx. 10 hours using a point by point mapping approach. FTIR imaging has allowed the collection of images in faster time with higher resolution. Moreover, no stray light problems that compromise spatial discrimination are involved (no aperturing), 
the spatial resolution is hardware/wavelength limited and the collection time is decoupled from the two for the imaged field of view. Typically, it is tougher to fabricate miniaturized detection systems and due to unique hardware issues, the performance of an FPA pixel typically lags behind single element mapping detectors that may be ten times larger. Thus, spectral (and image) quality desired usually determines the time for data acquisition.

The optical and infrared microscopic capabilities of an imaging instrument do not have to be matched. Thus, a major requirement of the mapping spectrometer that the optical and infrared paths be parfocal and collinear, is not necessary for imaging. The focusing in the sample plane can be independently carried out using a real time "bright field" IR image of the FPA. In practice, the optical path is not even required but an imaging spectrometer equipped with a field of view larger than the FPA camera helps to localize regions for imaging and examine neighboring regions before deciding to image them. Hence, most imaging instrumentation is equipped with a relatively cheap CCD visible camera with a field of view larger than that afforded by the more expensive FPA.

\section{2}

\section{Instrumentation}

At the core of most FTIR micro-imaging spectrometers is the step-scan interferometer [100]. The beam from the interferometer is diverted through standard microscope optics with the FPA at the end of the optical train. Cassegranian optics are used to focus radiation in the sample plane. Initially, a refractive setup consisting of an (usually $\mathrm{ZnSe}, \mathrm{BaF}_{2}$ or $\mathrm{CaF}_{2}$ ) imaging lens was used to form the image onto the FPA. This has given way to relatively aberration free reflective optics. The microscope setup is similar to that used in mapping experiments. A diffuser is sometimes used to increase spatial homogeneity in incident intensity [106]. The diffuser also reduces total light flux reaching the detector, thus preventing saturation of pixels. A bandpass filter can also be used to restrict wavelengths of light incident on the detector, which limits infrared flux to wavelengths of interest and eliminates background radiation outside it. An optimally sized cold shield inside the detector housing can be used to maximize available dynamic range by rejecting stray radiation arising outside the acceptance angle of the optics [101]. While the nominal resolution is determined by the system optics and the detector characteristics, the resolution limit is usually determined by the diffraction limit of the wavelength of interest.

A step-scan interferometer provides a means to maintain constant optical retardation for an arbitrarily large time. This large time is required when the FPA coadds collected frames at each optical retardation point to give reasonable signal to noise ratio images, which is similar to in-scan co-addition for regular step-scan spectroscopy. An electric pulse triggers each interferometer step, which may also 


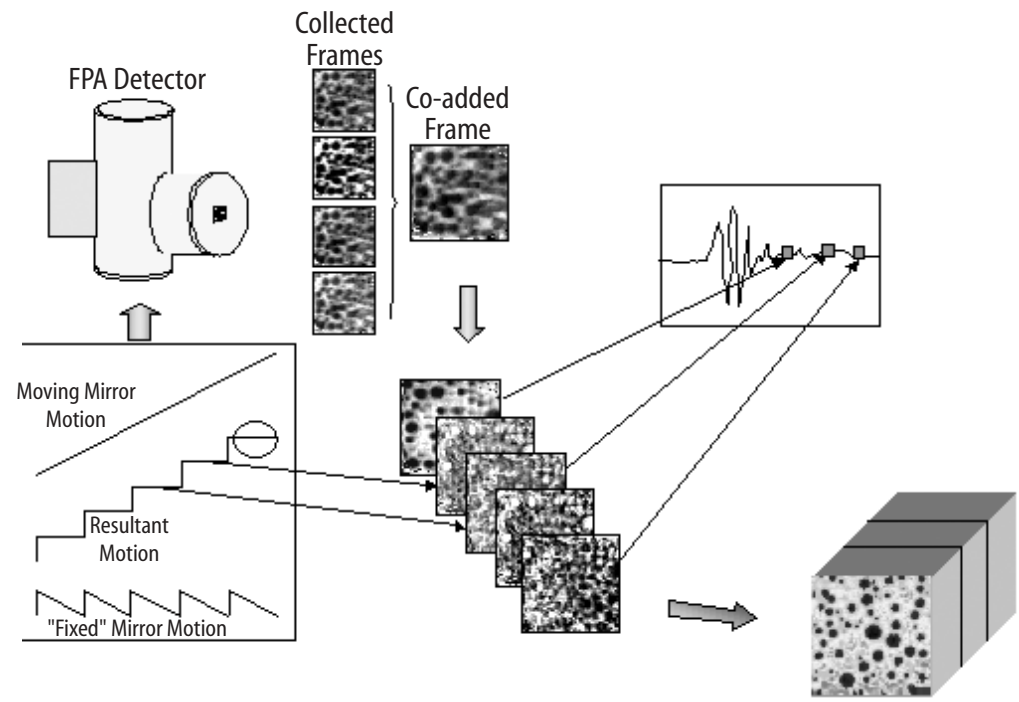

Fig. 10. Spectrometer Steps modulate the IR signal. At each step, the FPA collects frames and co-adds to yield a single frame for each step. Frames (equal to the number of steps) can be associated with a unique point on the interferogram. This interferogram data can be Fourier transformed, ratioed and truncated to yield an absorbance data cube

be used to initiate data acquisition by the FPA. A small delay to allow for mirror stabilization is allowed at the onset of the step and frames are then collected and some time is left for signal readout from the FPA and storage to a computer. The readout format may be sequential (common in MCT arrays) or single-shot (common in MCT and InSb arrays) in the focal plane. Details about solid state arrays [102] can be found elsewhere. The signal is integrated for a fraction of the time required for collection of each frame. The integration time, number of frames coadded and number of spectrometer steps (spectral resolution) determine the time required for the experiment and the data quality. This process is schematically shown in Fig. 10.

Another imaging configuration that utilizes a rapid scan interferometer has been proposed [103]. This configuration is essentially similar to a fast step-scan experiment in that the mirror speed is very low. Frames collected by the FPA are in a small enough time to be as close to instantaneous as possible on the time scale of the interferogram collection. However, the motion of the moving mirror does not allow co-addition of frames per interferometer retardation element. Hence, the process is faster but noisier. To minimize other sources of error contributing substantially to degrade the signal, the error arising from the deviation in mirror position during frame collection is sought to be made as small as possible. With current state of the art, this positional error is considerably larger than the error due 
to variations in mirror position during step-scan collection. The frame collection by the FPA (using a time interval based collection protocol) is triggered at the onset of the forward movement of the mirror. For maximal scanning speeds and to minimize positional errors, only one frame is collected. It is envisaged that this collected image can then be time-averaged with subsequently collected images similar to that of classical single detector element rapid scan data collection. The advantages lie in making the instrumentation cheaper than step-scan and when faster detectors are available, making data collection more efficient as the mirror stabilization time is not required [104].

\subsection{1}

\section{Sampling Techniques}

Sampling requirements and techniques are virtually identical to those for the single element spectrometer. However, spatial resolution achieved by using an array detector can range from less than $10 \mu \mathrm{m}$ to hundreds of kilometers. Hence, a wide variety of optical setups may be available for imaging purposes. Most polymeric materials examined to date have generally been examined using a microscope that images areas on the order of $\sim 500 \mu \mathrm{m} \times 500 \mu \mathrm{m}$ in transmission mode as this affords spatial resolution sufficient for many polymeric composites, a convenient optical setup and sufficient field of view for most instances. However, there is no fundamental reason why other types of optical configurations may not be employed for other length scales of examination. Some reflection experiments have been successfully conducted in our laboratories. However, reports on any studies utilizing reflectance configurations are yet to appear in the literature. Attenuated total reflection (ATR) imaging [105] and micro-ATR imaging are also being attempted by researchers. Improvements in the SNR attained in short experimental times by the evolving detector technology will likely benefit applications using these novel sampling techniques.

\subsection{2}

\section{Sources of Error}

FTIR imaging suffers from low signal to noise compared to other types of FTIR spectroscopy and microspectroscopy $[106,107]$. However, the major sources of error in an imaging system arise from detector noise and not from optical components of the imaging system (for example, apertures). The major factors in this regard are:

\subsubsection{1}

\section{Interferometer Noise}

Noise contributions from the interferometer arise for a variety of reasons. However, research grade spectrometers usually incorporate interferometers that have ex- 
cellent stability and low noise. Thus, the noise from the interferometer is usually small, especially when compared to noise from the detector. For step scan imaging instruments, noise due to the interferometer is almost three orders of magnitude lower than the noise due to the FPA.

\subsubsection{2}

\section{Detector Noise}

The small size of individual FPA detectors and their relatively early stage of development result in higher noise than usually observed in infrared detectors employed for spectroscopy. Read Noise in an FPA results from random charge movement in the array and its associated electronics. This is independent of the signal magnitude and hence, can be reduced by increased photon flux by an increase in signal or integration time. Read noise is usually reduced in detectors by using correlated double sampling, multiple pixel reads and binning [102].

Dark current noise usually forms the bulk of the detector noise. It results from accumulated charge in the detector. Impinging photons are not necessary to observe this noise and it can be detected as a signal in the FPA when radiation is blocked. It is usually due to thermal excitation of electrons to the valence band producing charges. Cooling the detectors considerably reduces this noise. Charge arising from dark currents increase linearly with time to reduce full well capacity resulting in poor dynamic range. However, it must be remembered that cooling also reduces the charge transfer efficiency. Given the desire for short experimental times and performance of IR sensitive FPAs, interferometer noise is usually orders of magnitude smaller than FPA noise and the noise arising in an imaging experiment can be considered entirely random.

\subsubsection{3}

\section{Failing Bump Bonds}

Most IR-sensitive arrays employed to examine the "fingerprint" region of the IR spectrum of polymers are hybrid arrays - an IR sensitive material is coated on top of an underlying FPA comprised of sophisticated readout circuitry. The first generations of IR sensitive arrays were made by attaching an IR sensitive material to a solid state array by conductive bumps. Repeated thermal cycling resulted in the failure of these bump bonds over time. The delaminating of pixels from their underlying substrate results in the pixels being inoperable and consequently, delaminated pixels appear as black squares on the IR image. Thus, spatial fidelity is compromised over time if a first generation array is used. Recently, IR sensitive materials have been grown using chemical techniques such as molecular beam epitaxy (MBE) on the underlying readout substrate. This has eliminated failing bonds and provided uniform arrays with a very small number of bad pixels. FPAs featuring bump-bonded substrates are unlikely to be employed for spectroscopic imaging in the future. 


\subsubsection{4}

Intensity Distribution, Pixel Saturation and Dynamic Range

Uneven intensity distribution leads to varying signal across the FPA field of view. While this is not a major concern when images are being ratioed and absorbance plotted, it is readily remediable. A simple array calibration (also termed flat fielding) before data collection is usually sufficient to provide a uniform field of view. However, individual pixel saturation needs to be avoided by reducing the flux at the detector, which may limit the signal at pixels with lower intensity. Pixel-to-pixel non-uniformity in the detector array is typically $\sim 5 \%$. Flat fielding prior to data collection also helps to reduce apparent heterogeneity due to manufacturing differences. For absorbance imaging applications, which are typical for polymeric systems, signal non-uniformity can be compensated for by ratioing against a suitable reference. The maximum dynamic range is determined by the A/D converter and is typically 14-16 bits for arrays in use today.

\subsubsection{5}

Sample

The source of the most unpredictable (and in most cases, un-correctable) noise pattern is the sample itself. Differences in refractive index at phase boundaries contribute to scattering at some pixels. This scattering at the interface results in an intensity loss at all frequencies leading to an offset in the baseline compared to other sample areas when sample single beams are ratioed to substrate single beams. While this deviation of the baseline from zero can be used to locate the interface without using a component specific absorbance frequency, it may cause problems in quantification [108]. The sample also reduces signal to noise ratio by reducing incident infrared intensity on the detector.

\subsection{3}

\section{Post-Collection Operations}

The major difference between all other forms of FTIR spectroscopy and imaging is the large volume of data generated in a short time period. Thousands of spectra with a specific location relative to each other need to be handled. This large amount of information may be organized in the form of a data cuboid, i.e., a threedimensional matrix. The first two dimensions denote the spatial location (i.e., specific pixel) while the third dimension stores the infrared spectrum of the sample imaged onto that pixel. Since the spectrum is digitized, the data cube may also be imaged to be a stack of two-dimensional arrays. The image corresponding to a particular data point (wavenumber), $\mathrm{z}_{\mathrm{i}}$, may then be extracted and the spatial distribution of the species with specific absorbance at that data point may be seen in the spatial area denoted by $\mathrm{E}\left(\mathrm{x}, \mathrm{y}, \mathrm{z}_{\mathrm{i})}\right.$. The extracted spectrum from a pixel at $\left(\mathrm{x}_{\mathrm{i}}, \mathrm{y}_{\mathrm{i}}\right)$ 


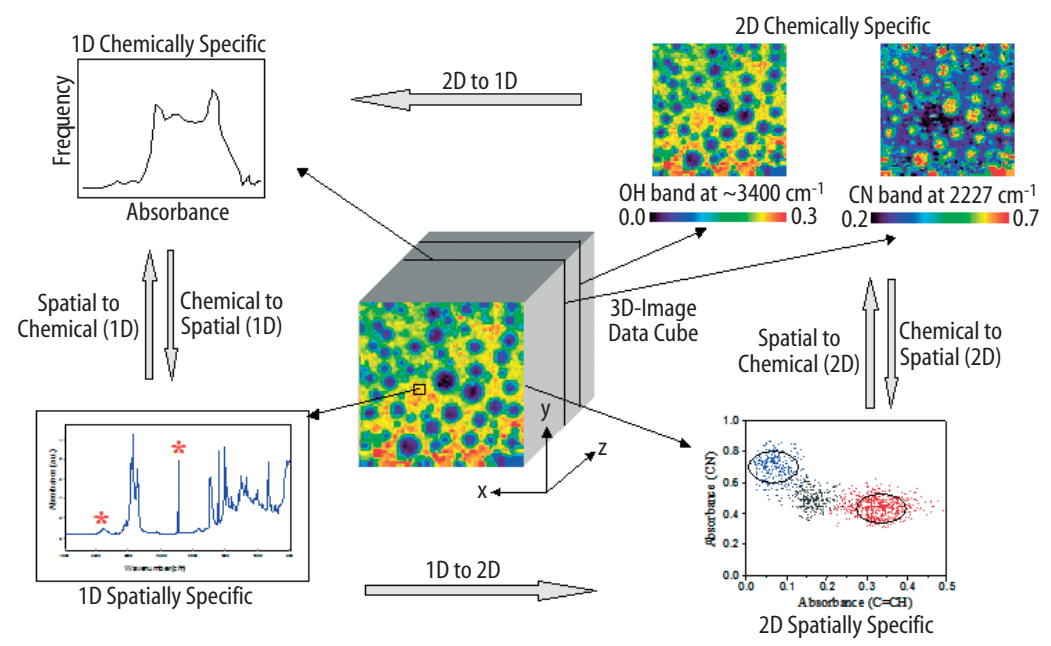

Fig. 11. Various visualizations possible with data extracted from the image data cube

may then be denoted as $\mathrm{E}\left(\mathrm{x}_{\mathrm{i}}, \mathrm{y}_{\mathrm{i}}, \mathrm{z}\right)$. The absorbance at a particular wavenumber at that pixel, $z_{i}$, can then be represented by $E\left(x_{i}, y_{i}, z_{i}\right)$.

The extraction of the image plane corresponding to a component specific wavenumber is the most popular method to visualize data. Images may be thresholded for maximum contrast between phases or between values for comparison across a set of images. Simple thresholding is affected by non-uniform thickness effects, which may lead to erroneous conclusions about a species' distribution. Peak height (or area) ratios are used to normalize thickness effects and relate the observed absorbance to species' concentration. The relative concentration of two species may be further seen using $2 \mathrm{~d}$ plots of the absorbance of one component against the other. The various visualizations possible are shown in Fig. 11. Clearly, the simplest form of representation to comprehend is a single number (zero dimensions) or a simple distribution plot ( 1 dimension). However, the information content is also reduced in such representations. Further, the whole image cube clearly contains all the informational content but is extremely difficult to visualize in its entirety and such a representation may not be completely relevant to the task. Hence, some form of trade-off between loss of relevant data and ease of representation is usually found in the analyses of an imaging experiment. The chart in Fig. 11 serves as a useful guide to some of the possibilities in such an endeavor.

The low SNR in FTIR images has been a major concern since the inception of the technique. The first effective approach had been to co-add as many frames as possible during the available experimental time. While this approach results in significant gains initially, the SNR as a function of co-added frames achieves a plateau [106]. Thus, after a number of frames, the benefits are relatively minor compared 


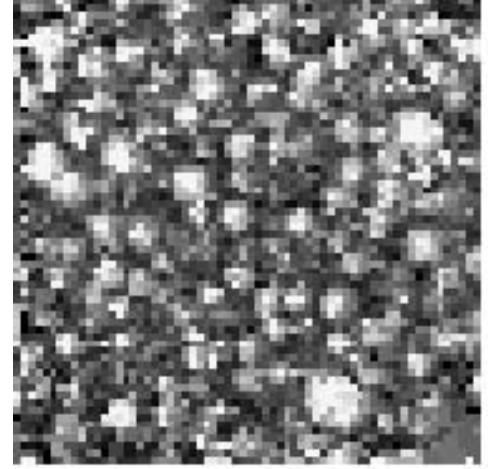

Single Image File

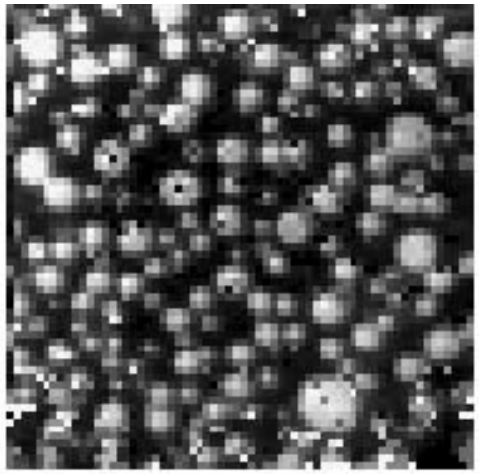

8 Image Files Co-added

Fig. 12. Noise reduction by co-addition as applied to a polymer dispersed liquid crystal sample. Co-addition results in a reduction of random salt and pepper noise allowing for better morphology visualization

to the large increase in experimental time. An optimum number may be derived based on the SNR as a function of co-added frames. Once the optimum number of co-added frames is determined, other techniques may be used to increase the SNR. One such technique has been to co-add single beam images of the sample and ratio them to similarly co-added single beam images used for background [106]. The SNR scales as $\sqrt{n}$, where $n$ is the number of sample single beam images co-added and ratioed against $n$ co-added background single beam images. The effects of this noise reduction procedure can be seen in Fig. 12. Random salt and pepper noise is vastly reduced allowing for better morphology visualization.

While image co-addition results in improved SNR characteristics, the process involves a $2 n^{2}$ increase in collection time for a SNR improvement factor of $n$. However, image co-addition is not suitable for application to real-time phenomena or fast examination of large numbers of samples. Another proposed idea was to ratio a single sample single beam image to multiple background images [109]. The resultant absorbance images are then co-added. This method, termed pseudo co-addition, is also limited in the benefits achievable, presumably because noise from the sample single beam starts to dominate the resultant. In many cases, the sample geometry will allow for the co-addition of many pixels with the same true absorbance [109]. This may be termed geometric co-addition (when co-added regions can be reproduced geometrically on the image) or phase co-addition (when pixels from a phase are selected with regard to the sample morphology). Spectra from these pixels can be extracted and co-added to yield a lower noise spectrum. The improvement in SNR scales as $\sqrt{n}$, where $n$ is the number of co-added pixels.

The benefits of pseudo co-addition are limited and the phase and geometric coadditions do not result in improved image quality. Mathematical noise reduction 

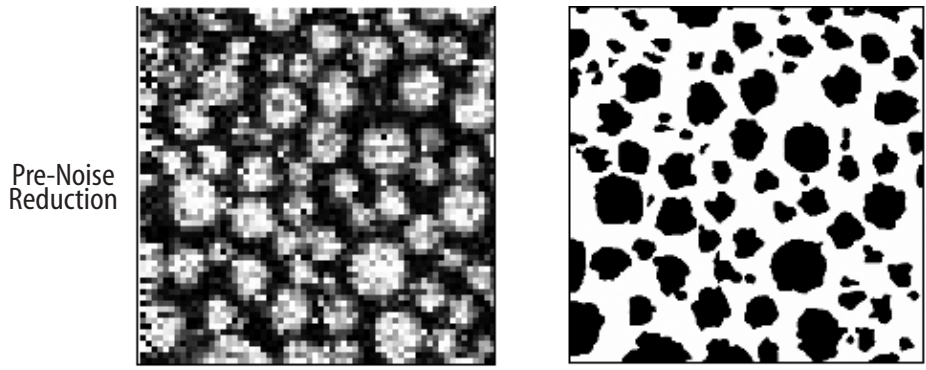

Post-Noise Reduction
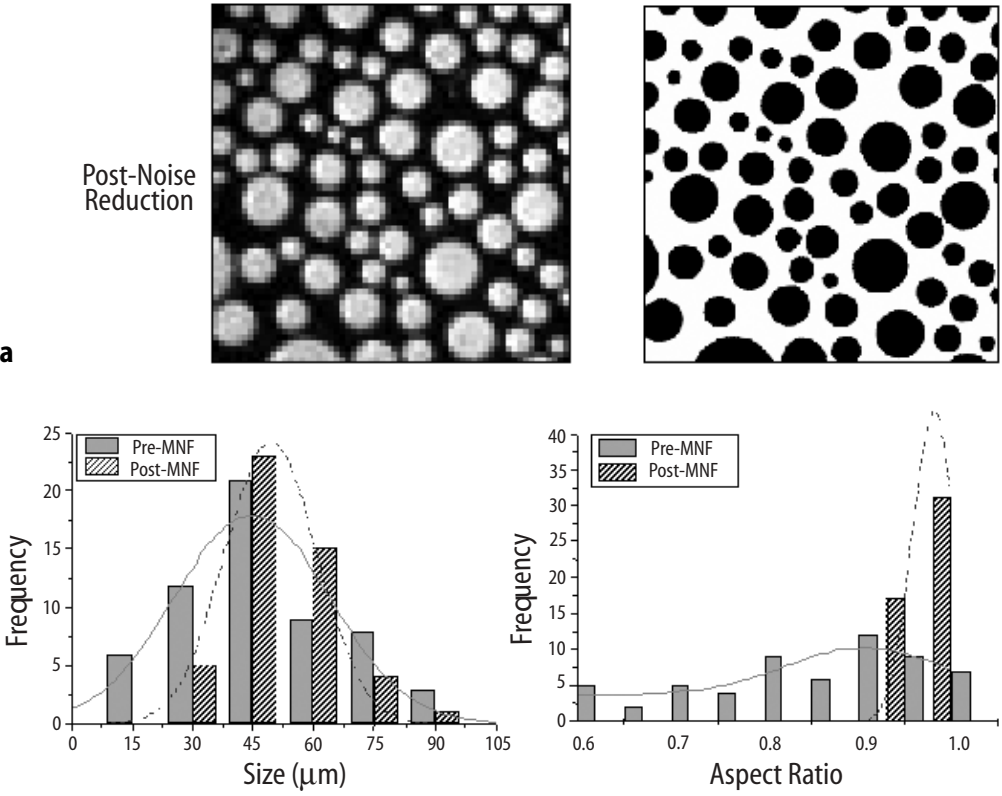

\begin{tabular}{|c|c|c|}
\hline Measure & Pre-MNF & Post-MNF \\
\hline Total Number of Domains & 85 & 75 \\
\hline Number of Complete Domains & 64 & 55 \\
\hline Average Major Axis (Hm) & 39.8 & 42.0 \\
\hline Average Minor Axis (Hm) & 34.0 & 41.5 \\
\hline Average Aspect Ratio & $0.82(0.09)$ & $0.96(0.02)$ \\
\hline Total Fractional Droplet Area & 37.7 & 41.8 \\
\hline
\end{tabular}

Fig. 13. (a) Binarized Images (right) for image analysis derived from (top, left) the original data, and (bottom, left) noise reduced data after application of the MNF transform to reduce noise (from Bhargava R, Wang S-Q, Koenig JL (2000) Appl. Spectrosc. 54: 486). (b) Morphology characteristic quantities derived from the binarized images for after image analysis (from Bhargava R, Wang S-Q, Koenig JL (2000) Appl. Spectrosc. 54: 486)

is an alternate pathway to higher fidelity images [110]. Mathematical techniques operating on the entire data set are computationally intensive but do not result in loss of the image or spectral content and do not affect collection time. A mathe- 
matical transform (the Minimum Noise Fraction [MNF] transform) that re-orders spectral data points into decreasing order of SNR was employed to transform images. Re-transforming the ordered data set using only a few relevant data points reduced noise. This approach is shown to result in significant gains in terms of image SNR (see Fig. 13). The actual gains were found to depend on the SNR characteristics of the original data. Noise is reduced by a factor greater than 5 if the noise in the initial data is sufficiently low. For a moderate absorbance level of 0.5 a.u., the achievable SNR by reducing noise is greater than 100 for a collection time of $\sim 3.5$ minutes. This noise reduction was shown to provide high quality images for accurate morphological analysis. The careful coupling of mathematical transformation techniques with spectroscopic FTIR imaging has tremendous potential to obtain high fidelity images without increasing collection time or drastically modifying hardware.

\section{3}

\section{Applications}

\subsection{1}

\section{Contaminants/Defects in Polymers}

The generally poor SNR characteristics of FTIR imaging limit its sensitivity to small concentrations. Recently, the SNR levels achievable for data collected in a matter of minutes have reached close to 1000 in a few minutes, implying quantification levels of a percent and detection levels down to a fraction of a percent in the same experiment. With the application of other co-addition and post-collection techniques, FTIR Imaging has the potential of being even more sensitive. Defects (such as air bubbles) in thin films may be easily visualized. An area where this technique is expected to be popular is the analysis of polymer inclusions and defects in the semi-conductor processing industry. The imaging of products will help determine the presence of contaminants and help in optimization of the process. With wide field imaging, even defects that have similar refractive indices with the examine material can be readily detected and separate visible imaging is not required.

There has been considerable controversy over the failure of breast implants and the subsequent contamination of breast tissue. Implants typically consist of an elastomeric shell with a soft filling inside. Over time and due to mechanical failure, the implant filling leaks into the breast tissue. This contamination may lead to various complications including capsular contracture, calcification, and some connective tissue disorders. Controversial aspects of the results of malfunction have not prevented numerous lawsuits. However, to assess any histopathological changes, it is first necessary to confirm the presence of material from the implant in tissue. The presence of silicone could be readily detected [111] in a tissue section using FTIR imaging. Si- $\mathrm{CH}_{3}$ characteristic vibrations were used to provide chemical 
contrast between the tissue and silicone inclusions as small as $\sim 10 \mu \mathrm{m}$. Inclusions could be readily found even in cases where optical microscopy contrast was poor.

\subsection{2}

\section{Amorphous Polymer Blends}

PVC is a useful polymer that is becoming less popular due to its degradation behavior. However, blends of PVC with some other polymers have the capability to yield composites with greater stability. In one such case, images of phase separated PVC/PMMA blends are reported [112]. Phase separation and concentration quantification can be carried out as a function of starting polymer properties and process conditions. However, the dispersion sizes examinable by infrared imaging are limited to tens of microns. In many polymeric systems, the dispersed phase is smaller than this dimension and the composites are not amenable to FTIR imaging analyses. Analyzing scattering [113] and relative concentrations of species in a pixel may yield qualitative clues to the morphology of phase separated material but probably cannot be used to obtain quantitative morphological information. We anticipate that spatial distribution of specific interactions in phase separated polymeric systems could also lead to such information and studies examining these aspects could appear in the near future.

\subsection{3}

\section{Semi-Crystalline Polymers and Their Blends}

FTIR imaging allows for the examination of semi-crystalline polymers via their dichroism $[98,114]$ The spherulitic structure, as visible in polarized optical microscopy, can be reproduced based on the orientation of transition dipole moments of functional groups in the sample. With an infrared polarizer in the beam path, the degree of orientation for each vibrational mode was determined by the generation of spatially resolved dichroic ratio images. When a melt-miscible polymer system is analyzed at temperatures lower than the crystallization temperature of one constituent, a phase separated or single crystalline phase structure might result. When the $T_{\mathrm{g}}$ of the non-crystalline component is lower than the crystallization temperature, the component phase separates as it is rejected from the crystal structure. The extent of phase separation may be easily visualized using IR imaging. PVAc/PEO and High $\mathrm{M}_{\mathrm{w}} /$ Low $\mathrm{M}_{\mathrm{w}}$ PEO blends have been imaged to examine the degree of segregation (Fig. 14) [114].

\subsection{4}

\section{Polymer Laminate Films}

The first reported application of FTIR imaging to polymeric materials was the imaging of a cross-section of a laminated polymer film. The adhesive layer between 

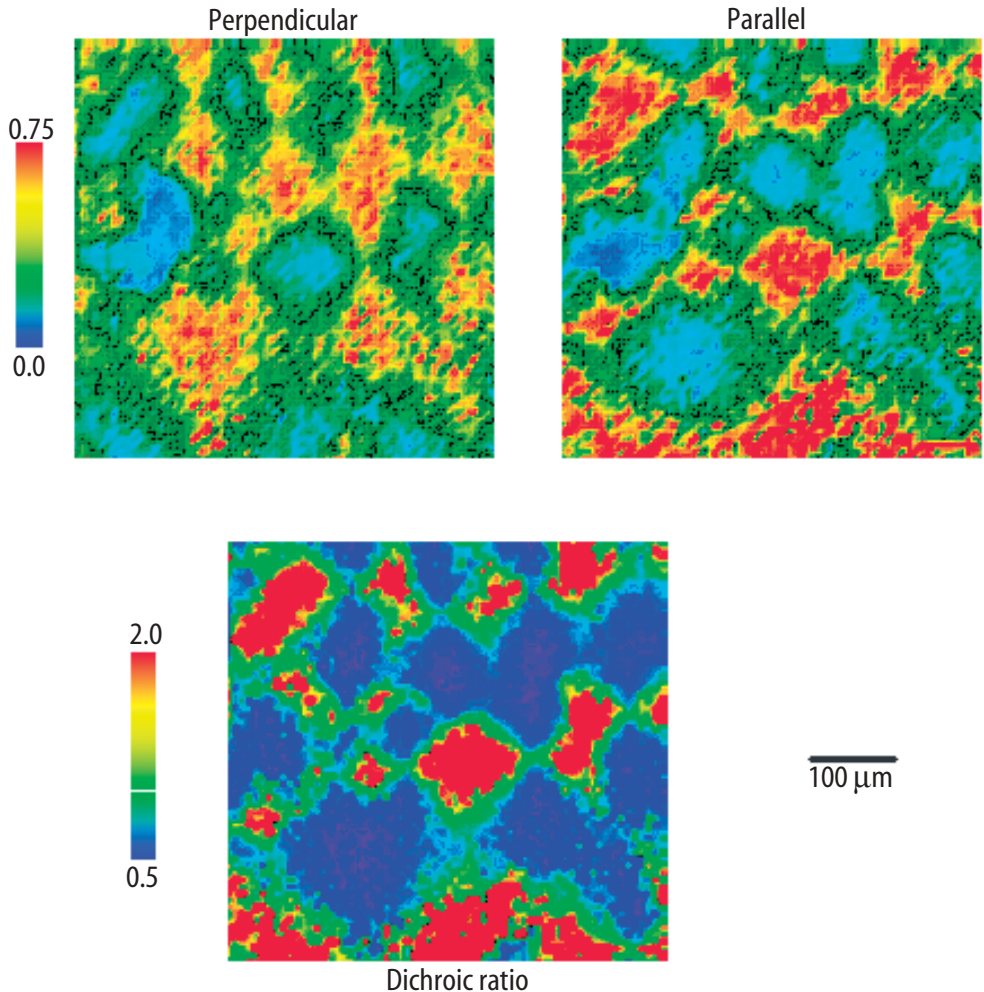

$\overline{100 \mu \mathrm{m}}$

Fig. 14. Infrared images of spherulites using perpendicular (left, top) and parallel (right, top) polarized radiation obtained from PEG $\left(\mathrm{M}_{\mathrm{v}}=35 \mathrm{~K}\right)$, quenched from $100^{\circ} \mathrm{C}$ to room temperature. The images were obtained by plotting the $1343 \mathrm{~cm}^{-1}: \mathrm{CH}_{2}$ wag vibrational mode of the PEG chain. The image at the bottom is a dichroic ratio image obtained by pixel-by-pixel division of the upper two. (reproduced from Snively CM, Koenig JL (1999) Spectroscopy)

two polymers was identified. Thin layers of PET, EVA and LDPE could be readily identified based on absorbance images from their characteristic vibrational peaks [115]. Since many properties (for example, barrier properties) of polymeric laminates depend on the structure of the composite, FTIR imaging presents a unique method to simultaneously examine the morphology and chemical composition of laminates for quality control. The morphology, composition and chemical interaction in the interphases can also be employed to correlate to properties and design better composite materials. High throughput chemical imaging as provided by FTIR imaging will considerably speed up quality checks and the characterization component of polymer engineering. 


\subsection{5}

\section{Polymer-Liquid Systems}

The dissolution of polymer films by liquids has been a subject of much investigation in polymer science. FTIR imaging presents a method to determine both spatial and spectral content of a polymer-solvent interphase. By monitoring the spatial distribution of concentration as a function of time from an initially known state, the diffusion of polymer and solvents as well as the dissolution rate can be determined. This experiment was among the first reported applications of FTIR imaging to real-time phenomena. The dissolution of a PMMA film was monitored and the polymer-solvent diffusion coefficient calculated [116]. In another study, it was shown that faster dissolution processes could also be monitored in real time using a combination of smaller collection times and co-addition processes [109]. The dissolution rate of the polymer film could then be obtained using its concentration profile as a function of time. The nature of the diffusion profiles can provide clues to the nature of the diffusion process. For example, a Case II behavior was found for MIBK diffusing into poly( $\alpha$-methyl styrene) (PAMS) [109].

Not only can dynamic phenomena in time but changes in liquid-polymer systems due to reaction can be measured readily. A polymer-liquid system consisting of phase-separated mixtures of uncured poly(Butadiene) and diallyl phthalate were studied to characterize morphology differences before and after the curing process [117]. Optical microscopy of these systems is particularly challenging as the resultant phases have similar refractive indices. However, good image contrast was achieved by FTIR imaging due to the inherent chemical differences manifest in their infrared spectra. The morphological changes were characterized over a period of time and the post-cure sample exhibited homogeneity at the resolution level of the instrument.

\subsection{6}

\section{Embedded Polymer Systems}

An area of interest for polymer applications is in the encapsulation of biologically active proteins in sustained release devices. Among these, microparticles composed of poly(lactic-co-glycolic acid)(PLGA) have been studied extensively [118]. As the capsule of biologic containing polymer is prepared, it is subjected to a number of manufacturing processes that may denature the biological molecule. In such a case, it is advantageous to have a method available to perform a spectroscopic analysis on the final product without the need to extract the biologic. In one such case, PLGA containing egg-lysozyme was analyzed using FTIR imaging [119]. Protein (Fig. 15) was found to be present inside the microspheres and distributed evenly on the scale of examination. Little protein was found on the surface and this result was confirmed by subsequent ATR and PAS measurements. This helped sug- 

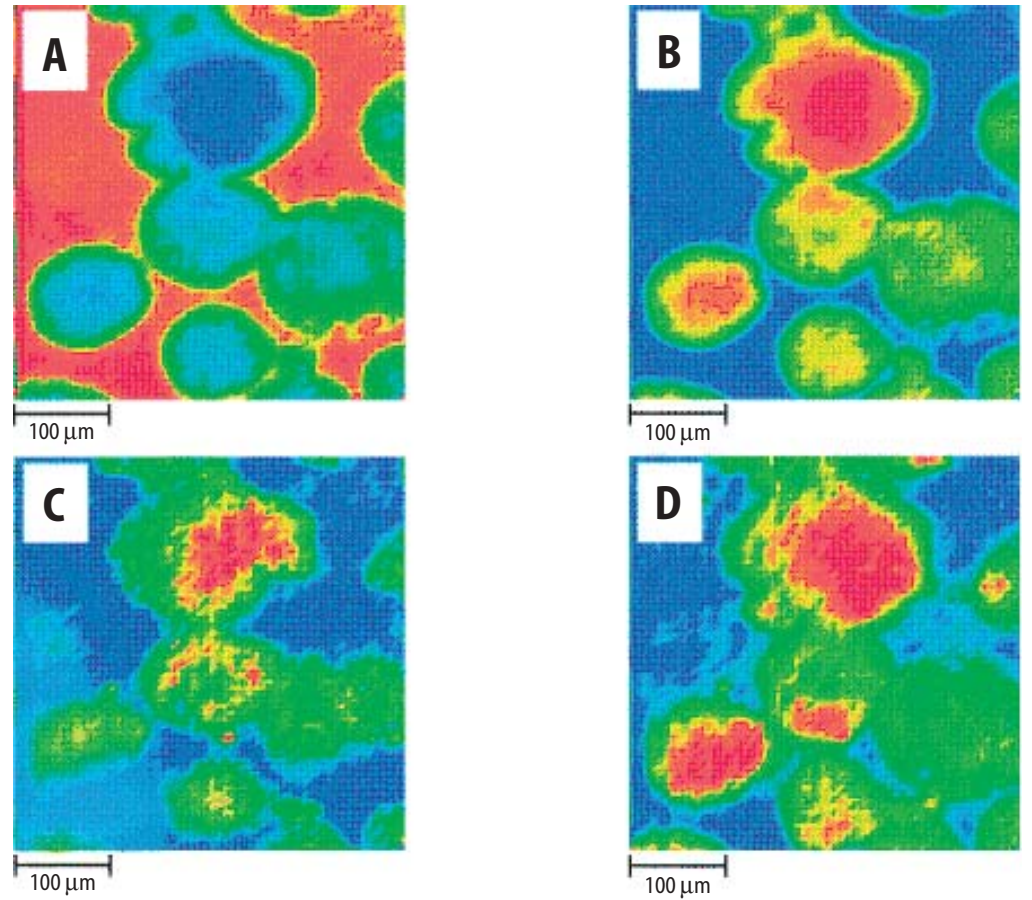

Fig. 15. False color infrared images of sliced lysozyme-loaded microspheres: blue indicates low intensity, green intermediate intensity, red high intensity. Intensity distribution of $\mathbf{A}$ embedding material, $1720 \mathrm{~cm}^{-1}$; B PLGA, $1756 \mathrm{~cm}^{-1}$; C protein Amide I, $1650 \mathrm{~cm}^{-1}$; D protein Amide II, $1550 \mathrm{~cm}^{-1}$. (reproduced from Ref. [119])

gest that an alternative mechanism of release from that proposed earlier $[120,121]$ may be at work. The formation and subsequent characterization of such composites can be carried out to not only examine the spatial distribution of chemical species afforded by examining but also obtain all the structurally sensitive information afforded by infrared spectroscopy. The structural changes and/or morphology development can then be characterized to the formation process providing a rapid analysis of the efficacy of the process.

\subsection{7}

\section{Polymer-Liquid Crystal Systems}

Polymer Dispersed Liquid Crystals (PDLCs) are widely researched materials of commercial importance in the electro-optical device industry as their light transmission can be changed by the application of an electric field. The refractive index of these rod-shaped liquid crystals along and perpendicular to their major axis is 


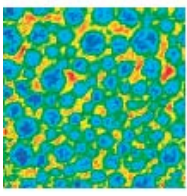

$5 \mathrm{~min}$

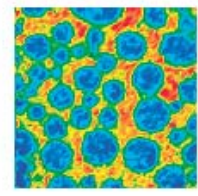

$10 \mathrm{~min}$

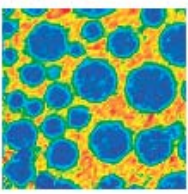

$15 \mathrm{~min}$

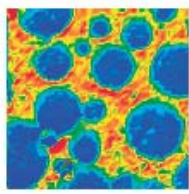

$30 \mathrm{~min}$

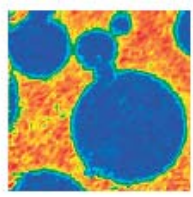

$90 \mathrm{~min}$
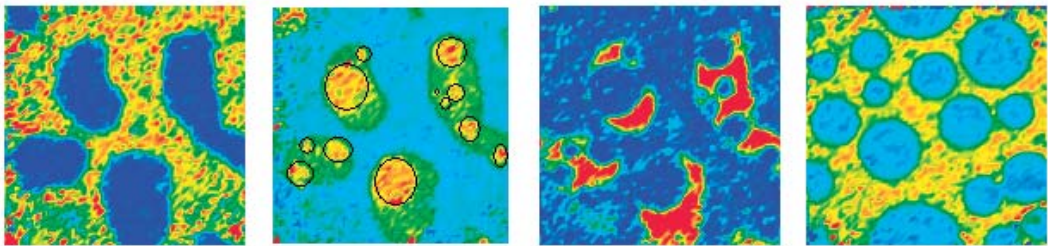

Fig. 16. The increase in droplet size can be seen as a function of time for a poly(butadiene) liquid crystal composite (top). The distribution of the polymer and the liquid crystal can be seen in the first two images (bottom), segregated as before. The surfactant (middle, right) can be seen to be distributed around the droplets and segregated from the matrix. A sample without surfactant

different. One of the refractive indices is chosen to correspond to that of the polymeric matrix in which they are to be imbedded. The preferred method for PDLC formation is to induce phase separation between the (growing) polymer and liquid crystal from their homogeneous solution. This method results in a liquid crystal phase with some matrix solubility and a matrix phase with high liquid crystal solubility. FTIR imaging of samples from a method used to lower this mutual solubility was carried out [122]. Since liquid crystals display phase sensitive IR responses [113], a calibration curve corresponding to the achieved concentrations was first constructed and the imaging data was subsequently used to determine concentrations. The concentration of each component in the other was calculated to a high degree of accuracy and the error estimated using statistical methods. The results from the imaging experiment agreed well with those from DSC and optical examination. FTIR imaging can also be used to visualize the distribution of surfactants in such systems [123]. The addition of surfactant was shown to retard droplet growth and was found to be close to the dispersed phase (liquid crystal) in the sample (Fig. 16).

The diffusion of liquid crystals into polymers is particularly intriguing given that the liquid crystal may be in an organized state or an isotropic state depending on the temperature. The diffusion of a liquid crystal (5CB) into a PBMA matrix was studied by using the contact method to prepare a gradient [114]. Concentration profiles were obtained as a function of time and temperature. The presence of an anomalous diffusion process was detected. It was shown that fast FTIR was able to correctly identify the diffusion process as anomalous. As opposed to this, a bulk 
mass uptake analysis would have led to the conclusion that the process proceeded according to Fick's second law. An abrupt change in the diffusion behavior was observed as a function of temperature. This change corresponded to the $T_{\mathrm{NI}}$ of the LC.

\subsection{8}

\section{Phase Separation in Polymers}

A tedious process is currently used to determine the phase diagram of binary polymeric systems. A single concentration is cycled through a temperature range and the onset of a refractive index difference (scattering or microscopy) is observed. Hence, a number of measurements are required at small temperature differences and accuracy depends on time spent at the experiment. This procedure is then repeated for many different concentrations to determine the phase diagram. Small refractive index (which may be temperature dependent) differences between the components may complicate analysis. Finally, the phase diagram may correspond to transitions in one (or both) components leading to a complicated situation. The basic issue in characterizing the phase separation behavior of a system is the determination of phase composition as a function of temperature. Any starting composition in the single phase region is capable of yielding information about two phases in the two-phase region if it phase separates upon changing the temperature to a region where the blend is non-homogeneous. If the chemical composition of the phases is directly determined, say by FTIR imaging, then the phase diagram can be obtained.

FTIR imaging coupled to thermal control of the sample [124] has been employed to determine phase compositions. We next report a study where the phase diagram of a liquid crystal mixture, E7, in a polymer (PBMA) was obtained. A single composition $(80 \% \mathrm{LC})$ is held in the single phase region and quenched to different temperatures in the two phase region. Once hydrodynamic and coarsening equilibrium is attained, the sample is imaged (Fig. 17a). We acquired images continuously over time and used data from an image only when the spatial features were larger than $\sim 20 \mu \mathrm{m}$ and the structures of two consecutive images were found to not change. The characteristics absorbances are determined and phase composition obtained from a previously determined calibration curve. Thus, the two points of the co-existence curve at the temperature of quench are simultaneously determined. In this manner, the compositions at each temperature are determined. The phase diagram (Fig. 17b) obtained corresponds well with the phase diagram obtained using optical microscopy following the classical methods of obtaining phase diagrams. Using the described approach, phase diagrams may be readily obtained in a fast, straightforward manner that is decoupled from the kinetics of the phase separation process. However, there is a discrepancy between the optical and infrared predictions, which needs to be further explored. 


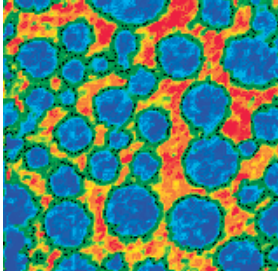

$23^{\circ} \mathrm{C}(0.0-1.5)$

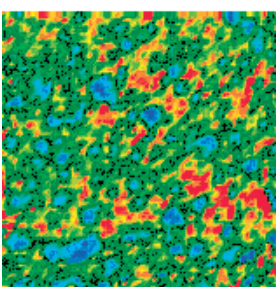

a

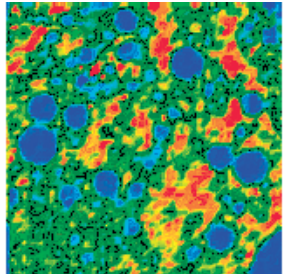

$32^{\circ} \mathrm{C}(0.0-1.3)$

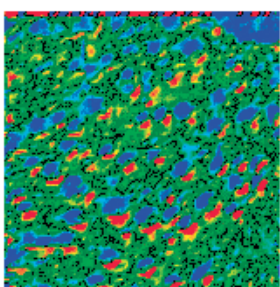

$43^{\circ} \mathrm{C}(0.0-0.9)$

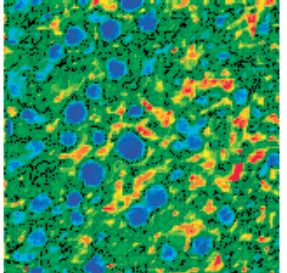

$35^{\circ} \mathrm{C}(0.0-1.15)$

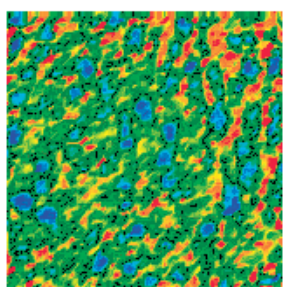

$53^{\circ} \mathrm{C}(0.0-0.7)$

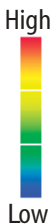

$100 \mu \mathrm{m}$

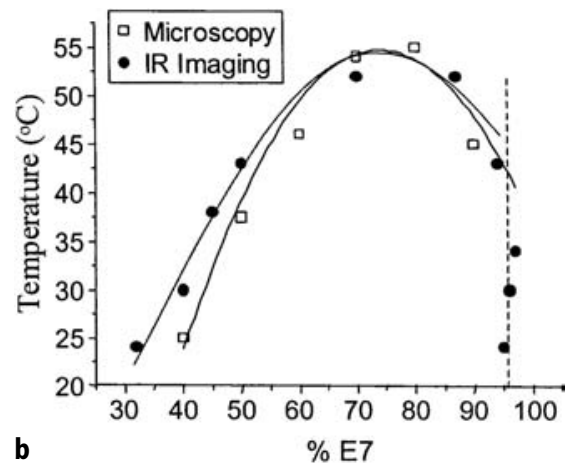

Fig. 17. (a) A liquid crystal mixture, E7, dispersed in PBMA (80\% liquid crystal by weight) imaged after quenching from the single phase to various temperatures. The figures in parenthesis indicate the low and high limits the images are plotted between as shown by the color bar on the right. The characteristics infrared spectroscopic absorbance from the two phases can be determined to yield concentrations from a previously obtained calibration curve (not shown). (b) The phase diagram of the liquid crystal mixture, E7, dispersed in PBMA imaged in Fig. 16(a) compared to the phase diagram obtained by optical microscopy reported in Ref. [84]

\section{4}

\section{Summary}

FTIR imaging using an FPA coupled to an interferometer has been demonstrated as an excellent technique to achieve non-invasive spatially specific information. It 
combines the benefits of an IR spectrometer with those of an optical microscope providing unprecedented amount of information and level of detail in both the spectral and spatial domains. Some unique considerations are required in the collection and processing of the large amount of data from an imaging spectrometer. In a short time, FTIR imaging has been applied to a number of multicomponent polymer samples with good results. The techniques used for and applications to multicomponent polymers are expected to increase exponentially in the foreseeable future as the cost of instrumentation reduces and the SNR characteristics of available detectors improve.

\section{4}

\section{Other Infrared Microspectroscopy Approaches}

\section{1}

\section{Hadamard Transform Infrared Microscopy}

Hadamard transform infrared microscopy involves the use of encoding masks [125] to achieve selective light throughput from the optical train. By using masks of known patterns or encoding, a large spatial area could be mapped to provide an effective multichannel advantage. Spatial multiplexing of the field of view can also be accomplished by the use of masks while retaining the advantages of the Fourier transform [126]. The process is schematically shown in Fig. 18. While this tech-

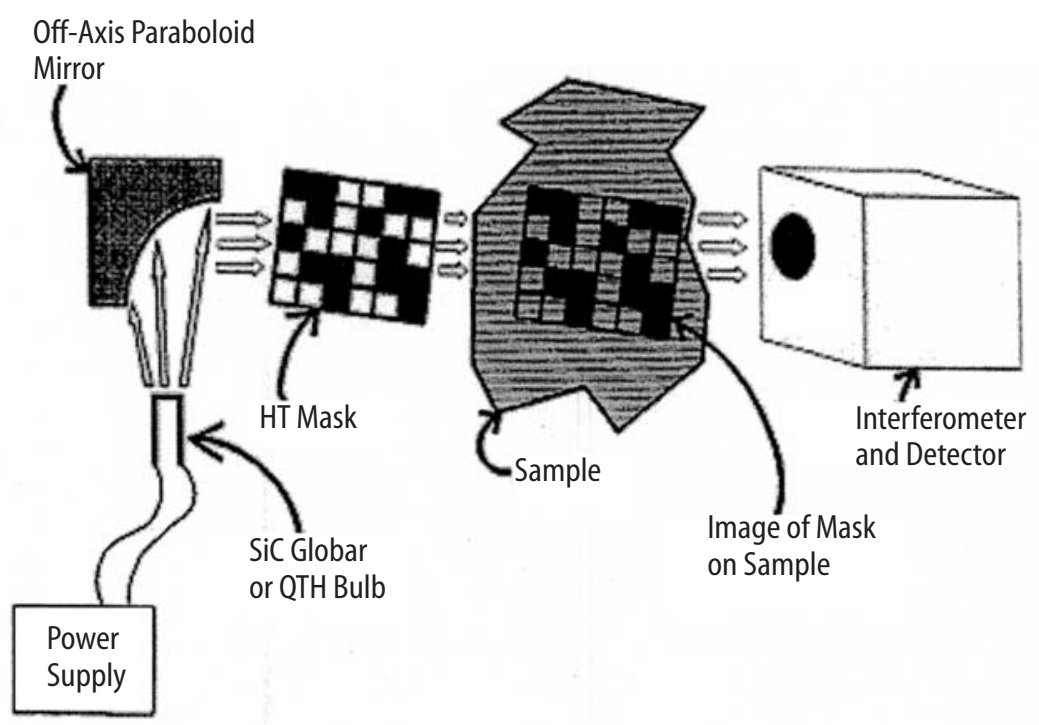

Fig. 18. Schematic of the chemical mapping instrumentation in the Mid- and Near-IR Spectral Regions by Hadamard Transform/FT-IR Spectrometry. (Reproduced from [126]) 
nique is not new, it has also not achieved as widespread applicability as the single aperture microscopy technique. The primary limitation of the technique arises from its need for opaque masks, which need to be reproducibly and rapidly positioned. Recently, programmable micro-mirror arrays have been used to achieve large multichannel advantages compared to raster scan approaches [127]. Wide applicability of this route to conduct microspectroscopy will determine on the advances in optical masking that will probably evolve from optical communications applications in the future.

\section{2}

\section{Synchrotron Infrared Microscopy}

The large collection times and poor SNR for high spatial resolutions in IR microscopy are largely a result of relatively weak light intensity. A synchrotron source provides for very high throughput and it's coupling to an infrared microscope results in high quality data. Not only is the spatial resolution of an infrared microscope using a synchrotron source superior and SNR higher ( 100 times) compared to a standard globar source, it also provides an extended spectral range. Hence, data collection over wide spectral ranges is considerably faster and sensitivity for thin samples is excellent. Apertures as low as $3 \times 3 \mu \mathrm{m}^{2}$ have been used [128]. However, the major drawback arises from the unique component of the technique: a synchrotron source is required. Thus, reports in literature examining polymeric materials have been limited [129] as the instrument is not widely available.

\section{3}

\section{Solid State Focal Plane Array Imaging}

It is also possible to use infrared acousto-optic tunable Filters (AOTFs) [130] to restrict radiation on the array to be of a certain wavelength and sequentially sample different wavelengths using multichannel detection by employing array detectors [131]. Fig. 19 demonstrates an application [132], in which plastic waste was classified using numerical methods from data collected using remote and on-line near-infrared measurements on a macroscopic scale using a focal plane array. In a variation of the approach of using filters in conjunction with array detectors [133], an acousto-optic tunable filter (AOTF) was employed to diffract unpolarized incident light into two diffracted beams with orthogonal polarization. One of the beams was directed to an array detector sensitive in the visible region (a silicon camera) while the other is detected to an infrared sensitive FPA. Near-IR spectroscopy could be carried out while the visible image was simultaneously observed. A curing epoxy-amine system was found to exhibit spatial inhomogeneities when examined by the instrument. Such an arrangement, however, introduces polariza- 
(a) Geometric Material Information

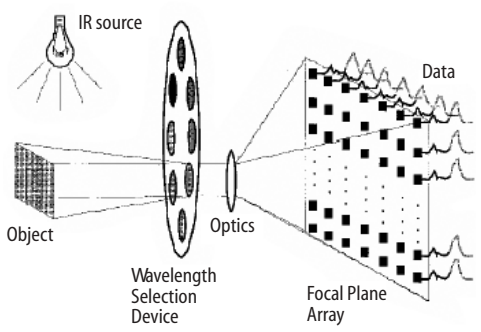

(b)

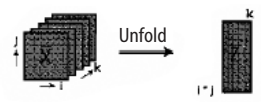

Model Building (Calibration)

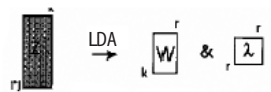

RE-Classification

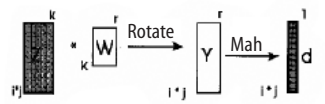

Prediction of Unknowns

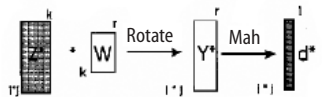

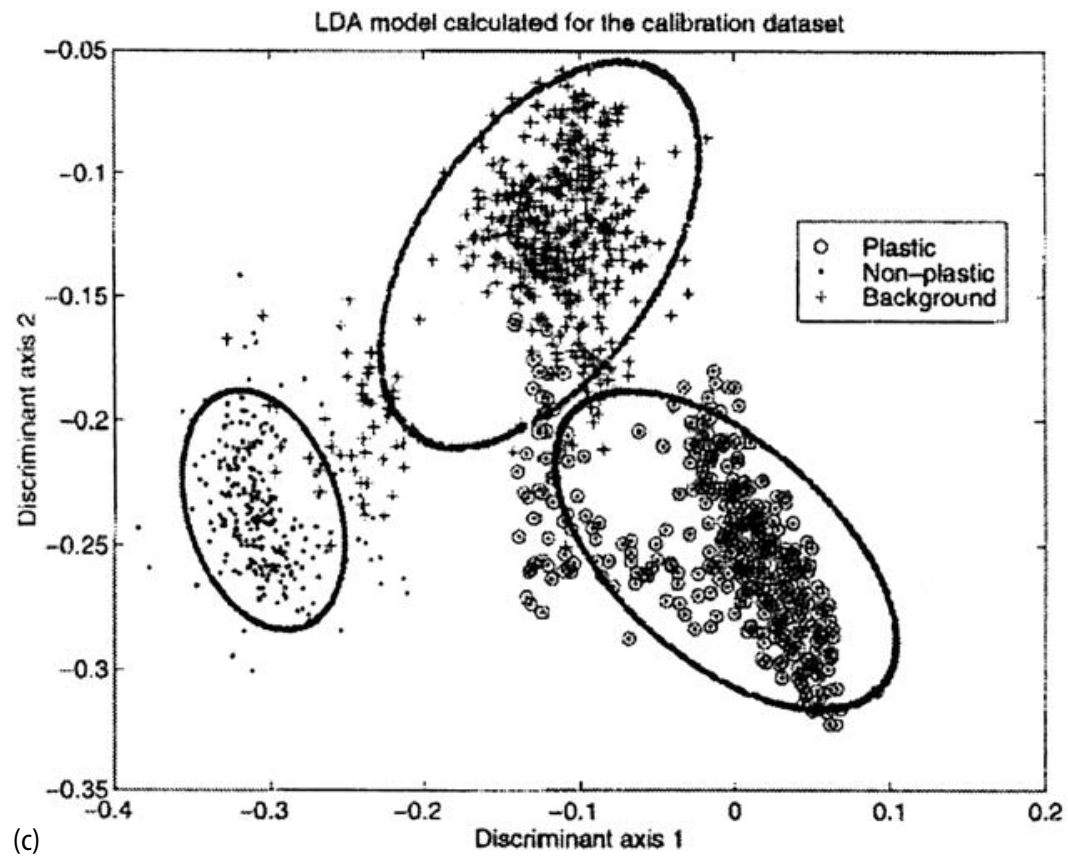

Fig. 19. (a) Schematic view of the measurement principles. Reflected radiation from an object is measured for several wavelength regions. Each individual detector pixel integrates possible reflected photons in the particular wavelength range. (b) Schematic representation of the LDA classification procedure. The three-dimensional calibration dataset $\mathbf{X}$ is unfolded to a two-dimensional matrix $\mathbf{Z}$, which is used to calculate weight matrix $\mathbf{W}$. Next, matrix $\mathbf{Z}$ is transformed to $\mathbf{Y}$ where for each class (nc) the covariance matrix and corresponding means are calculated, according to the Mahalanobis measure. An unknown image $\mathbf{Z}^{*}$ is transformed to $\mathbf{Y}^{*}$ and classified to $\mathbf{d}^{*}$ with the use of the calculated covariance and mean data. (Reproduced from Ref. [132]). (c) Visualization of the calibration dataset using two linear discriminant components for three classes: plastic, nonplastic, and background mini-spectra. The ellipses indicate the classes modeled by LDA. In this manner, waste is discriminated 
tion effects in the spectra. In many oriented polymer systems, these polarization effects may be useful to determined relative organization of sub-molecular species, especially for thick polymer films.

Yet another approach to spatially resolved infrared spectroscopy using a focal plane array can be visualized where one of the dimensions of the focal plane is employed to detect spectrally discriminated data. The combination of a device that disperses different wavelengths to different spatial locations and an FPA can be employed to image a line perpendicular to the wavelength dispersion direction [134]. Alternately, the same can be achieved by means of a linear variable filter (LVF). In either case, the spatial position and wavelength discrimination are linked. Hence, an integrated system needs to be designed where the sample is moved such that every pixel samples a different set of a wavelength and a spatial component to yield the entire imaging data set. These approaches have not been employed for imaging yet, but can be expected to be available in the near future for at least some special analysis cases. While the optical components in these configurations are fixed, resulting in a cheaper and more robust system, the sample would need to be moved.

Clearly, solid state approaches such as these provide an alternative where high spatial fidelity is required, a high rate of data acquisition is desirable and a rugged system required. The optical arrangement determines the spatial resolution of the system, which can range from the usual "macroscopic" configuration where the FPA is employed for multichannel detection or a combination of the optical system with a sophisticated data analysis protocol is for spatially resolved data collection. While solid state approaches are useful in such settings and provide flexibility and rapid data acquisition, interferometer based systems provide much higher sensitivity, broad spectral coverage, and make possible much higher spectral resolutions.

\section{4}

\section{Scanning Probe Microspectroscopy or Photothermal Imaging}

A new technique is under development combining scanning probe microscopy at sub-micron spatial resolution with FTIR spectroscopy. The idea springs from a recently developed technique termed Micro-Thermal Analysis [135, 136]. This technique allows localized thermal analysis to be combined with surface and sub-surface imaging by means of scanning thermal microscopy. Chemical fingerprinting is thereby achieved, giving a microscopic version of two well-known thermal analysis techniques, TMA and modulated-temperature DSC. It also provides for combining the advantages of the thermal characterization techniques with infrared spectroscopy.

Modulated radiation from a standard spectrometer is directed onto a sample and detected by a microprobe (see Fig. 20) [137, 138]. The IR imager's thermal probe is the same as used in micro-thermal techniques. It detects photo-thermal response of a region on the specimen heated by exposure to the beam from a FTIR 


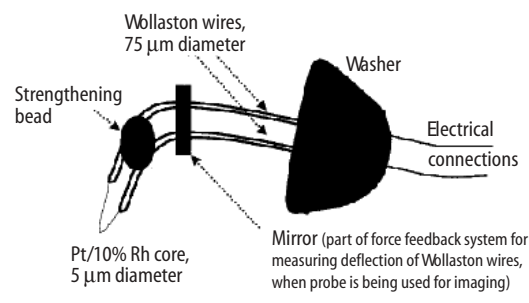

(a)

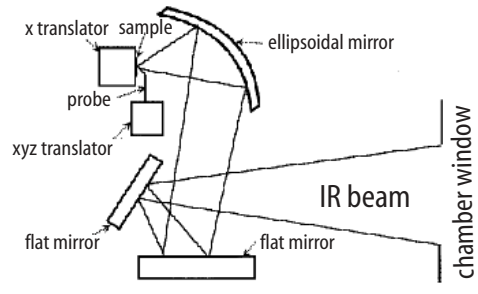

(b)

Fig. 20. a Schematic Diagram for a thermal probe and $\mathbf{b}$ schematic diagram of the probe and sample arrangement layout inside the spectrometer. (Reproduced from Ref. [137])

spectrometer. The signal from this probe measures the resulting temperature fluctuations, thus provides an interferogram that replaces the interferogram normally obtained by means of direct detection of the IR transmitted by a sample. This enables IR microscopy at a spatial resolution below diffraction limits as the resolution now depends on the probe and not system optics. This resolution could be as low as tens of nanometers. However, the interaction of the probe with the sample may not be desirable and may limit the actual resolution achieved.

\section{5}

\section{Near Field Infrared Mapping}

Near-field infrared microscopy allows examination of spatial regions at a spatial resolution better than the diffraction limit imposed by the wavelength of the probing light. One approach employs the use of a standard FTIR interferometer-microscope combination [21]. The experimental setup was such that near-field conditions were possible but the effective spatial resolution attained was not clear. The In another implementation, the diffraction limit is overcome by employing specialized radiation collection optics for the infrared region [139] that permit partial conversion of the evanescent fields into propagating waves. The amount of light propagating from a sub-wavelength aperture through a flat substrate strongly increases, as the distance between the light collection tip and the sample is decreased. Hence, for a sample, topographic artifacts often dominate near-field images, which are sought to be eliminated by flat sample preparation techniques [140]. In addition, the transmitted power is dependent on the refraction index of the sample, resulting in deviations between the near-field and far-field spectra. The most useful applications for the apertureless near-field IR spectroscopic techniques will involve ideal sample preparation, multiplexing of signal, complementary information from mathematical modeling and better probe design [141]. Near-field systems are expected to be especially useful for imaging phase-separated high polymer blends, sub-microscopic self-organization, thin films and aqueous polymer systems. 


\section{6 \\ Infrared Diode-Based Imaging}

An alternative approach to using interferometers for imaging is to use another source that provides spectral discrimination directly. One such source is an infrared diode laser. A laser coupled to an infrared microscope provides a means to rapidly obtain spatial resolution approaching the diffraction limit [142]. A multi-layer polymer composite contaminated by migration of a volatile additive from an exogenous source was imaged using this system. The distribution of this additive in the layered structure was shown to correlate with specific layers (Fig. 21). A con-

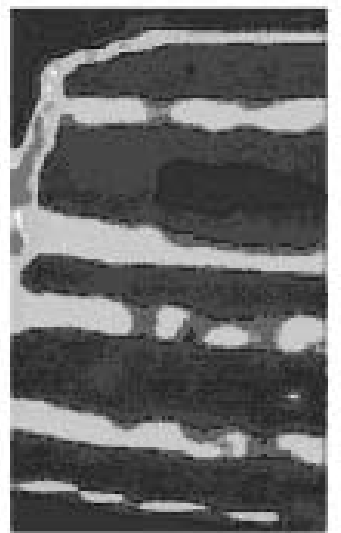

C

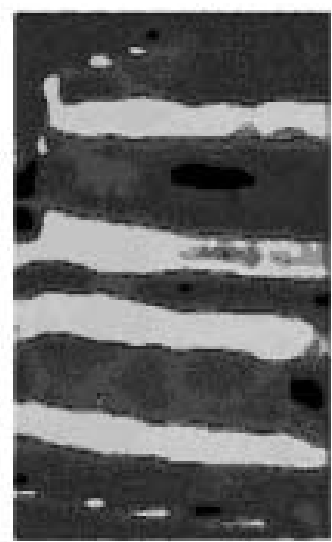

b

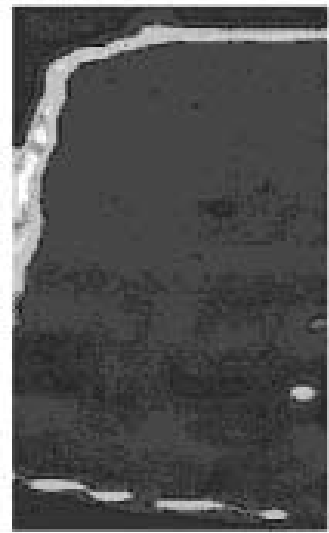

$\mathrm{d}$

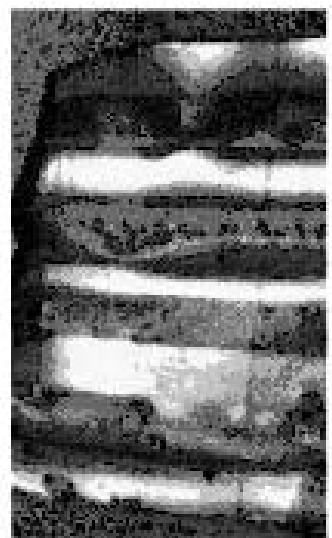

Fig. 21. Diode laser false-color images of an NP-exposed sample: $\mathbf{a}$ absorbance at $1568 \mathrm{~cm}^{-1}$, b absorbance at $1530 \mathrm{~cm}^{-1}$, and $\mathbf{c}$ difference between (a) and (b). A white-light image is shown in (d). The absorbance intensity maps to the color scale adjacent to each image. Each image is $300 \mu \mathrm{m} \_500 \mu \mathrm{m}$ (Reproduced from Ref. [142]) 

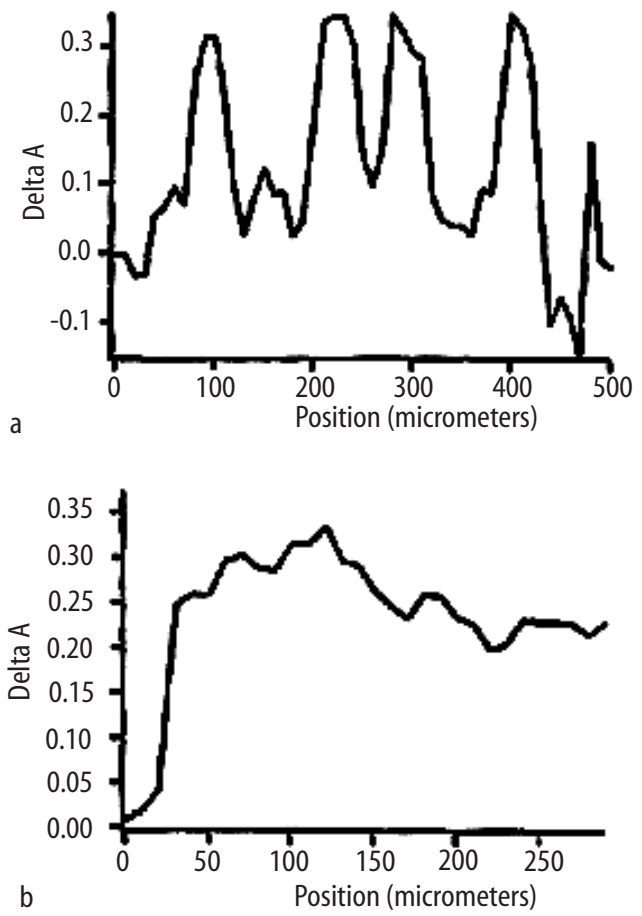

Fig.22. Absorbance profiles from diode laser images in Fig. 21. a $100 \mu \mathrm{m}$ from left edge of image (perpendicular to layer structure); b $100 \mu \mathrm{m}$ from top edge of image (parallel to layer structure following an epoxy-binder layer)

centration gradient suggesting a diffusive mechanism of additive migration parallel to the layered structure was observed (Fig. 22). A coupling of the laser to a focal plane array could provide a multichannel advantage, considerably increasing the scope of such instrumentation.

\section{7}

\section{Summary}

There are a number of approaches to allow infrared microspectroscopic investigations of polymers. While interferometer based approaches dominate the instrumentation in general, other approaches may be effective in specific cases or when dedicated instrumentation examining a limited range of problems is desired. FPAs, employed to achieve spatial discrimination as in FTIR imaging or spatial and spectral discrimination as in dispersive and filter approaches, provide flexibility and faster spectroscopy due to their multichannel detection advantage. Many new approaches to microspectroscopy are dedicated to achieving spatial resolution better 
than limited by diffraction. While the progress to that end is remarkable, most techniques are far from developed to a mature enough level to be routinely applied. It is expected that the maturation of these technologies and the resulting instrumentation will be particularly useful for examining the interfacial regions in polymeric samples or cases where domains are smaller than the resolution limit of current techniques. However, the SNR characteristics would probably require large collection times for a single image and may result in the instruments being used for specialized cases where current imaging methods are inapplicable. The development of new imaging capabilities can only mean greater progress for the scientist studying multicomponent polymers.

\section{5}

\section{Concluding Remarks}

\section{1}

\section{Instrumental Directions}

The trend towards more stable spectroscopic systems with high spatial resolution and lower spectral noise data acquired in smaller time periods is going to be a feature of instrumental development for many years to come. This new generation of instruments awaits the development of more sensitive, faster response and lower noise detectors as well as better miniaturized electronics. As problems in polymer science become more sophisticated, the need to analyze samples with greater precision, track changes in a sample more minutely and carry these out faster is going to be a primary need for the spectroscopist. New accessories continue to be developed for old techniques and several specialized accessories will no doubt be available for the emerging techniques in the near future. New instrumentation consisting of motionless spectrometers, ultrafast spectrometers and sub-micron resolution imaging spectrometers will probably develop in the foreseeable future. In particular, FTIR imaging techniques have reached maturity levels where they can be used for routine analysis and we expect to see a large increase in the applications reported. Development of larger format arrays with expanded wavelength sensitivity and higher efficiency is one direction, which will spur such growth. Emergence of faster and more sophisticated computers continues to help in data acquisition and analysis.

\section{2}

\section{Applications}

We believe that much growth in applications will be spurred on by the desire of scientist to observe dynamic phenomena and to analyze large numbers of samples in a short time period. Static, spatially resolved measurements have been routinely carried out for over 30 years. The last decade has seen a major initiative towards 
examining dynamic phenomena ranging from simple changes like polymerization to complicated spatially resolved interactions. Even physical phenomena ranging from orientation to phase separation to temperature rise continue to be explored. Such demanding/specialized needs will contribute to further development. A major area where spatially resolved methods will become popular is in the analysis of combinatorial libraries [143]. The multichannel detection advantage of the imaging techniques available today is well suited to the analysis of high throughput reaction sequences and is expected to be widely applied in this field in the future.

\section{3}

\section{Processing Techniques and Strategies}

FTIR spectroscopy has been applied to identify many multicomponent systems using techniques such as spectral subtraction and factor analysis. The high degree of reproducibility and accuracy of IR measurements have made this possible. One of the largest reasons for that has been the use of digital computers. Indeed the development of spectroscopy has gone hand in hand with that of faster, cheaper and better computing power. High spectrometer fidelity and reproducibility is possible not only because of better hardware but numerous checks and control loops for fine corrections that can be made using sophisticated computers. The very existence of mapping and imaging technologies is due to the ability to handle large data sets made possible by computers.

It may be envisioned that, in the coming years, appropriate software will play an increasing role in the collection, post-collection data extraction and manipulation, identification of species and processing of results. Multivariate analysis coupled to automated detection schemes and fuzzy logic identification protocols based on spectroscopic data will be increasingly used. Automated Artificial Neural Networks coupled to FTIR instrumentation [144] can then be used to monitor and control the formation and properties of multicomponent polymers. To make that aim possible, numerical methods for the analysis and characterization of polymers [145] are becoming increasingly important. The use of imaging techniques in synthesis, processing and quality control applications is expected to grow with the development of stable and ruggedly usable instrumentation.

\section{4}

\section{Summary}

FTIR microspectroscopy has made remarkable progress in understanding multicomponent polymer systems. A range of issues - from static morphology to submolecular molecular dynamics can now be analyzed by the versatile set of tools this technique affords. The last 5-10 years have been a period of intense development on both instrumental and data interpretation fronts. For applications to pol- 
ymers, a lot of effort has been devoted to understand interactions between polymers, dynamics of polymers and microscopic characterization of polymers. These analyses have been carried out faster and with greater accuracy than ever before. The future holds good promise for the development in spatially resolved infrared spectroscopic techniques, especially imaging, which will greatly assist polymer scientists in structure-property relationship elucidations.

\section{References}

1. Painter PC, Coleman MM, Koenig JL (1982) The theory of vibrational spectroscopy and its application to polymeric materials. John Wiley, New York

2. Koenig JL (1992) Spectroscopy of polymers. American Chemical Society (ACS), Washington DC, USA

3. Chalmers JM, Everall NJ, Hewitson K, Chesters MA, Pearson M, Grady A, Ruzicka B (1998) Analyst 123:579

4. Smith MJ, Carl RT (1989) Appl. Spectrosc. 43:865

5. Barer R, Cole ARH, Thompson HW (1949) Nature 163:198

6. A general discussion of sampling techniques for microscopy may be found in Allen TJ (1992) Vib. Spectrosc. 3:217

7. Augerson CC (1998) Appl. Spectrosc. 52:1353

8. Tungol MW, Bartick EG, Montaser A (1993) Appl. Spectrosc. 47:1655

9. Lang PL, Katon JE, Schiering DW, O’keefe JF (1986) Polym . Mat. Sci. Eng. 54:381

10. Kaito A, Nakayama K (1992) Macromolecules 25:4882

11. Harrick, NJ, Milosevic M, Berets SL (1991) Appl. Spectrosc. 45:944

12. Gentner JM, Wentrup-Byrne E (1999) Spectrochim. Acta. A-Mol. Bio. 55:2281

13. Lewis L., Sommer AJ (1999) Appl. Spectrosc. 53:375

14. Mirabella FM Jr, (1987) Applications of microscopic Fourier transform infrared spectrophotometry sampling techniques for the analysis of polymer systems. In: Rousch PB (ed) The design, sample handling and applications of infrared microscopes. American Society for Testing and Materials, Philadelphia, PA, USA, p 74

15. Ferrer N (1997) Mikrochim Acta 329

16. Reffner JA; Ressler G; Schiering DW; Wihlborg WT (1997) Mikrochim Acta 333-337

17. Blair D.S., Ward KJ (1991) Part. Surf. 3:123

18. Sommer AJ, Katon, JE (1991) Appl. Spectrosc. 45:1663

19. Chase B (1998) In: Messerschmidt RG, Hartcock MA (eds) Infrared microspectroscopy. Marcel Dekker, New York, USA, p 97

20. Messerschmidt RG (1987) In: Rousch PB (ed) The design, sample handling and applications of infrared microscopes. American Society for Testing and Materials, Philadelphia, PA, USA p 12

21. Sahlin JJ. Peppas NA (1997) J. Appl. Polym. Sci. 63:103

22. Born M, Wolf E (1980) Principles of optics, 6th edn. Pergamon, Elmsford NY, USA

23. Heimann PA, Urstadt R (1990) Appl. Opt. 29:495

24. Koenig JL (1992) Microspectroscopy of polymers. ACS, Washington, DC, USA

25. Katon JE (1994) Vib. Spectrosc 7:201

26. Rintoul L, Panayiotou H, Kokot S, George G, Cash G, Frost R, Bui T, Fredericks P (1998) Analyst 123:571

27. Cournoyer R, Shearer JC, Anderson DH (1977) Anal Chem 49:2275

28. Hartcock MA (1987) In: Rousch PB (ed) The design, sample handling and applications of infrared microscopes. ASTM STP 949. American Society for Testing and Materials, Philadelphia, PA, USA, p 85 
29. Shearer JC, Peters DC (1987) In: Rousch PB (ed) The design, sample handling and applications of infrared microscopes. American Society for Testing and Materials, Philadelphia, PA, USA, $\mathrm{p} 27$

30. Weesner FJ, Carl RT, Boyle RM (1991) Proc SPIE Int Soc Opt Eng, 1575:486

31. Ward KJ (1989) Proc SPIE Int Soc Opt Eng 1145:212

32. Garcia D (1997) J Vinyl Additive Tech. 3:126

33. Hartcock MA, Lentz LA, Davis BL, Krishnan K (1986) Appl Spectrosc 40:

34. Joshi NB, Hirt DE (1999) Appl Spectrosc 53:11

35. Gavrila DE, Gosse B (1994) J Rad Nuc Chem 185:311

36. Jouan X, Gardette JL (1987) Polym Commun 28:239

37. Miseo EV, Guilmette LW (1987) Industrial problem solving by microscopic Fourier transform infrared spectrophotometry. In: Rousch PB (ed) The design, sample handling and applications of infrared microscopes. ASTM STP 949. American Society for Testing and Materials, Philadelphia, PA, USA, p 97

38. Wilhelm $P$ (1996) Micron 27:341

39. Ahlblad G, Forsstrom D, Stenberg B, Terselius B, Reitberger T, Svensson LG (1997) Polym Degrad Stabil 55:287

40. Tungol MW, Bartick EG, Montaser A (1990) 44:543

41. Grieve MC; Griffin RME; Malone R (1998) Sci Justice 38:27

42. Bouffard SP, Sommer AJ, Katon JE, Godber S (1994) Appl Spectrosc 48:1387

43. Katon JE, Lang PL, Schiering DW, O'Keefe JF (1987) Instrumental and sampling factors in infrared microspectroscopy. In: Rousch PB (ed) The design, sample handling and applications of infrared microscopes. ASTM STP 949. American Society for Testing and Materials, Philadelphia, PA, USA, p 4

44. Bartick EG(1987) Considerations for fiber sampling with infrared microspectroscopy. In: Rousch PB (ed) The design, sample handling and applications of infrared microscopes. ASTM STP 949. American Society for Testing and Materials, Philadelphia, PA, USA, p 64

45. Cho LL, Reffner JA, Gatewood BM, Wetzel DL (2001) J Forensic Sci 46:1309

46. Chase DB (1987) Infrared microscopy: a single fiber technique. In: Rousch PB (ed) The design, sample handling and applications of infrared microscopes. ASTM STP 949. American Society for Testing and Materials, Philadelphia, PA, USA, p 4

47. Hirschfeld T (1985) Appl Spectrosc 39:424

48. see for example, Cho LL, Reffner JA, Wetzel DL (1999) J Forensic Sci 44:283

49. Vermette P, Thibault J, Levesque S, Laroche G (1999) J Biomed Mater Res 48:660

50. Costa L, Luda MP, Trossarelli L, del Prever EMB, Crova M, Gallinaro P (1998) Biomaterials 19:659

51. Kakoulides EP, Smart JD, Tsibouklis J (1998) J Control Release 54:95

52. Kressler J, Schafer R, Thomann R (1998) Appl Spectrosc 52:1269

53. Benedetti E, Catanorchi S, D’Alessio A, Vergamini P, Ciardelli F, Pracella M (1998) Polym Int 45:373

54. Ludvigsson M, Lindgren J, Tegenfeldt J (2000) J Electrochem Soc 147:1303

55. Withey RE, Hay JN (1999) Polymer 40:5147

56. Karamancheva I, Stefov V, Soptrajanov B, Danev G, Spasova E, Assa J (1999) Vib Spectrosc 40:369

57. Camacho NP, Rinnerthaler S, Paschalis EP, Mendelsohn R, Boskey AL, Fratzl P (1999) Bone 25:287

58. Benedetti E; Catanorchi S; D’Alessio A; Vergamini P; Ciardelli F; Pracella M (1998) Polym Int 45:373

59. Dibbern-Brunelli D, Atvars TDZ, Joekes I, Brabosa VC (1998) J Appl Polym Sci 69:645

60. Schafer R, Zimmermann J, Kressler J, Mulhaupt R (1997) Polymer 38:3745

61. Coleman PB, Ramamurthy AC (1999) Appl Spectrosc 53:150

62. Gal T, Toth P (1992) Can J Appl Spectrosc 37:55

63. Kellner R, Fischböck G, Minich C (1986) Mikrochim Acta 1:271

64. Nishioka T, Nakano T, Teramae N (1992) Appl Spectrosc 46:1904 
65. Pell RJ, Mckelvy ML, Hartcock MA (1993) Appl Spectrosc 47:634

66. Guilment J, Markel S, Windig W (1994) Appl Spectrosc 48:320

67. High MS, Painter PC, Coleman MM (1992) Macromolecules 25:797

68. Sahlin JJ, Peppas NA (1997) J Biomat Sci Polym Ed 8:421

69. Sheu K, Huang S J, Johnson JF (1989) Polym Eng Sci 29:77

70. Hsu SC, Lin-Vien D, French RN (1992) Appl Spectrosc 46:225

71. Cameron RE, Jalil MA, Donald AM (1994) Macromolecules 27:2713

72. Riquet AM, Wolff N, Laoubi S, Vergnaud JM, Feigenbaum A (1998) Food Addit Contam 15:690

73. Schafer R, Kressler J, Neuber R, Mulhaupt R (1995) Macromolecules 28:5037

74. Iijima M, Ukishima S, Iida K, Takahashi Y, Fukada E (1995) Jap J Appl Phys Lett 34:L65

75. Kaito A, Kyotani M, Nakayama K (1992) Polymer 33:2672

76. Glime J; Koenig JL (2000) Rubber Chem Technol 73:47

77. Kaito A, Kyotani M, Nakayama K (1993) J Polym Sci B Poly Phys 31:1099

78. Kaito A, Kyotani M, Nakayama K (1993) J Appl Polym Sci 48:2147

79. Fondeur F, Koenig, JL (1993) Appl Spectrosc 47:1

80. Fondeur F, Koenig, JL (1993) J Adhes 43:263

81. Palinko I, Torok B, Prakash GKS, Olah GA (1998) Appl Catal A-Gen 174:147

82. Ohtake Y, Kobayashi T, Asabe H, Murakami N (1998) Polym Degrad Stabil 60:79

83. Challa SR, Wang S-Q, Koenig JL (1996) Appl Spectrosc 50:1339

84. Challa SR, Wang S-Q, Koenig JL (1997) Appl Spectrosc 51:297

85. Wall BG, Koenig JL (1998) Appl Spectrosc 52:1377

86. Challa SR, Wang S-Q, Koenig JL (1997) Appl Spectrosc 51:10

87. McFarland CA, Koenig JL, West JL (1993) Appl Spectrosc 47:321

88. McFarland CA, Koenig JL, West JL (1993) Appl Spectrosc 47:598

89. Kohri S, Kobayashi J, Tahata S, Kita S, Karino I, Yokoyama T (1993) Appl Spectrosc 47:1367

90. Markwell RD, Butler IS, Gao JP, Shaver A (1992) Appl Org Chem 6:693

91. Maverich AM, Ishida H, Koenig JL (1995) Appl Spectrosc 49:354

92. Arvanitopoulos C, Koenig JL (1996) Appl Spectrosc 50:1

93. Arvanitopoulos C, Koenig JL (1996) Appl Spectrosc 50:11

94. Noobut W, Koenig JL (1999) Polym Composite 20:38

95. Dalton S, Heatley F, Budd PM (1999) Polymer 40:5531

96. Lewis EN, Treado PJ, Reeder RC, Story GM, Dowrey AE, Marcott C, Levin IW (1995) Anal Chem 67:3377

97. Colarusso P, Kidder LH, Levin IW, Fraser JC, Arens JF, Lewis EN (1998) Appl Spectrosc 52:106A

98. Koenig JL, Snively CM (1998) Spectroscopy 13:22

99. Bhargava R, Wall BG, Koenig JL (2000) Appl Spectrosc 54:470

100. Lewis EN, Kidder LH, Arens JF, Peck MC, Levin IW (1997) Appl Spectrosc 51:563

101. Bhargava R, Fernandez DC, Schaeberle MD, Levin IW (2000) Appl Spectrosc 54:1743

102. Harnly JM, Fields RE (1997) Appl Spectrosc 51:334A

103. Snively CM, Katzenberger S, Oskarsdottir G, Lauterbach J (1999) 24:1841

104. Huffman SW, Bhargava R, Levin IW (2002) Appl Spectrosc 56:965

105. Sommer AJ, Tisinger LG, Marcott C, Story GM (2001) Appl Spectrosc 3:252

106. Snively CM, Koenig JL (1999) Appl Spectrosc 53:170

107. Bhargava R, Levin IW (2001) Anal Chem 73:5157

108. Bhargava R, Wang S-Q, Koenig JL (1998) Appl Spectrosc 52:323

109. Bhargava R, Ribar T, Koenig JL (1999) Appl Spectrosc 53:1313

110. Bhargava R, Wang S-Q, Koenig JL (2000) Appl Spectrosc 54:1690

111. Kidder LH, Kalasinsky VF, Luke JL, Levin IW, Lewis EN (1997) Nature Med 3:235

112. Artyushkova K, Wall BG, Koenig JL, Fulghum JE (2000) Appl Spectrosc 54:1549

113. Bhargava R, Wang S-Q, Koenig JL (2000) Macromolecules 32:8989

114. Snively CM, Koenig JL (1999) J Poly Sci Pol Phys 37:2353 
115. Marcott C, Story GM, Dowrey AE, Reeder RC, Noda I (1997) Mikrochim Acta Suppl 14:157

116. Snively CM, Koenig JL (1998) Macromolecules 31:3753

117. Oh SJ, Koenig JL (1998) Anal Chem 70:1768

118. van de Weert M, Hennink WE, Jiskoot W (2000) Pharma Res 17:1159

119. van der Weert M, van't Hof R, van der Weerd J, Heeren RMA, Posthuma G, Hennink WE, Crommelin DJA (2000) J Control Release 68:31

120. Cohen S, Yoshioka T, Lucarelli M, Hwang LH, Langer R (1991) Pharm Res 8:713

121. Takahata H, Lavelle EC, Coombes AGA, Davis SS (1998) J Control Release 50:237

122. Bhargava R, Wang S-Q, Koenig JL (1999) Macromolecules 32:2748

123. Koenig JL,Wang S-Q, Bhargava R (2000) Inst Phys Conf Ser 165:43

124. Koenig JL, Wang S-Q, Bhargava R (2001) Anal Chem 73:351A

125. Zhang F, Gu T (1990) Proc SPIE 1205:150

126. Bellamy MK, Mortensen AN, Hammaker RM, Fateley WG (1997) Appl Spectrosc 51:477

127. DeVerse RA, Hammaker RM, Fateley WG (2000) Appl Spectrosc 54:1751

128. Guilhaumou N, Dumas P, Carr GL, Williams GP (1998) Appl Spectrosc 52:1029

129. Ocola LE, Cerrina F, May T (1997) Appl Phys Lett 71:847

130. Bennet JM (1976) Appl Opt 15:2705

131. Lewis EN, Levin IW (1995) J Microscopy Soc Am 1:35

132. Van den Broek WHAM, Wienke D, Melssen WJ, Feldhoff R, HuthFehre T, Kantimm T, Buydens LMC (1997) Appl Spectrosc 51:856

133. Tran CD (2000) J Near Infrared Spectrosc 8:87

134. Elmore DL, Tsao MW, Frisk S, Chase DB, Rabolt JF (2002) Appl Spectrosc 56:145

135. Hammiche A, Reading M, Pollock HM, Song M, Hourston DJ (1996) Rev Sci Instrum 67:4268

136. Pollock HM, Hammiche A, Song M, Hourston DJ, Reading M (1998) J Adhesion 67:217

137. Hammiche A, Pollock HM, Reading M, Claybourn M, Turner PH, Jewkes K (1999) Appl Spectrosc 53:810

138. Anderson MS (2000) Appl Spectrosc 54:349

139. Piednoir A, Licoppe C, Creuzet F (1996) Optics Commun 129:414

140. Palanker DV, Simanovskii DM, Huie P, Smith TI (2000) J Appl Phys 88:6808

141. Dragnea B, Leone SR (2001) Int Rev Phys Chem 20:59

142. Bailey JA, Dyer RB, Graff DK, Schoonover JR (2000) Appl Spectrosc 54:159

143. Snively CM, Oskarsdottir G, Lauterbach J (2000) J Comb Chem 2:243

144. Dolmatova L, Ruckebusch C, Dupuy N, Huvenne J-P, Legrand P (1998) Appl Spectrosc 2:329

145. Chalmers JM, Everall NJ (1996) Trends Anal Chem 15:18

Editor: J. E. McGrath

Received: June 2002 